\title{
Efficacy of Interventions to Improve Respiratory Function After Stroke
}

\author{
Kênia KP Menezes PT PhD, Lucas R Nascimento PT PhD, Patrick R Avelino PT MSc, \\ Maria Tereza Mota Alvarenga, and Luci F Teixeira-Salmela PT PhD
}

\author{
Introduction \\ Methods \\ Focused Questions \\ Identification and Selection of Trials \\ Participants, Interventions, and Outcome Measures \\ Quality and Risk of Bias \\ Data Analysis \\ Results \\ Flow of Trials Through the Review \\ Characteristics of the Included Trials \\ Effect of Interventions \\ Discussion \\ Conclusion
}

BACKGROUND: The aim of this study was to systematically review all current interventions that have been utilized to improve respiratory function and activity after stroke. METHODS: Specific searches were conducted. The experimental intervention had to be planned, structured, repetitive, purposive, and delivered with the aim of improving respiratory function. Outcomes included respiratory strength (maximum inspiratory pressure $\left[\mathbf{P}_{\text {Imax }}\right]$, maximum expiratory pressure $\left[\mathbf{P}_{\text {Emax }}\right]$ ) and endurance, lung function (FVC, $\mathrm{FEV}_{1}$, and peak expiratory flow [PEF]), dyspnea, and activity. The quality of the randomized trials was assessed by the PEDro scale using scores from the Physiotherapy Evidence Database (www.pedro.org.au), and risk of bias was assessed in accordance with the Cochrane Handbook for Systematic Reviews of Interventions. RESULTS: The 17 included trials had a mean PEDro score of 5.7 (range 4-8) and involved 616 participants. Meta-analyses showed that respiratory muscle training significantly improved all outcomes of interest: $P_{\text {Imax }}$ (weighted mean difference $11 \mathrm{~cm} \mathrm{H} \mathrm{H}_{2} \mathrm{O}, 95 \%$ CI 7-15, $\left.\mathrm{I}^{2}=0 \%\right), \mathrm{P}_{\mathrm{Emax}}\left(8 \mathrm{~cm} \mathrm{H} \mathrm{H}_{2} \mathrm{O}, 95 \%\right.$ CI 2-15, $\left.\mathrm{I}^{2}=65 \%\right)$, FVC $\left(0.25 \mathrm{~L}, 95 \%\right.$ CI $\left.0.12-0.37, \mathrm{I}^{2}=29 \%\right), \mathrm{FEV}_{1}\left(0.24 \mathrm{~L}, 95 \%\right.$ CI $\left.0.17-0.30, \mathrm{I}^{2}=0 \%\right)$, PEF $(0.51 \mathrm{~L} / \mathrm{s}$, 95\% CI $0.10-0.92, \mathrm{I}^{2}=0 \%$ ), dyspnea (standardized mean difference -1.6 points, 95\% CI -2.2 to $-0.9 ; I^{2}=0 \%$ ), and activity (standardized mean difference $0.78,95 \%$ CI $0.22-1.35, I^{2}=0 \%$ ). Meta-analyses found no significant results for the effects of breathing exercises on lung function. For the remaining interventions (ie, aerobic and postural exercises) and the addition of electrical stimulation, meta-analyses could not be performed. CONCLUSIONS: This systematic review reports 5 possible interventions used to improve respiratory function after stroke. Respiratory muscle training proved to be effective for improving inspiratory and expiratory strength, lung function, and dyspnea, and benefits were carried over to activity. However, there is still no evidence to accept or refute the efficacy of aerobic, breathing, and postural exercises, or the addition of electrical stimulation in respiratory function. Key words: stroke; spirometry; maximal respiratory pressures; comparative effectiveness research; dyspnea; motor activity. [Respir Care 2018;63(7):920-933. (c) 2018 Daedalus Enterprises] 


\section{Introduction}

Stroke is the second-leading cause of death globally and the leading cause of disability worldwide. ${ }^{1}$ Previous studies have demonstrated that stroke affects not only the muscles of the upper and lower limbs, but also those of the respiratory system. ${ }^{2,3}$ Individuals with stroke typically demonstrate changes in breathing pattern, ${ }^{4}$ decreased ventilatory function, ${ }^{5}$ decreased strength of the respiratory muscles, ${ }^{2,6}$ and reduced activity of the paretic diaphragm. ${ }^{7,8}$ In addition, decreased respiratory function is associated with deconditioning, activity limitations, and elevated risk for respiratory complications. ${ }^{9}$ Disabilities of the respiratory system after stroke associated with dysphagia and ineffective cough may increase the risks of aspiration pneumoniae, which has been described as the leading cause of nonvascular death after stroke. ${ }^{10}$ Thus, implementing interventions that have the potential to improve respiratory function and, consequently, to prevent morbidity and mortality in people with stroke is vindicated. ${ }^{11,12}$

Respiratory function is related to the breathing process, in which the lungs perform their function of ventilation and perfusion, and, thus, properly oxygenate all body tissues. ${ }^{13}$ However, this process depends on proper functioning of all the involved structures, such as suitable strength and endurance of the respiratory muscles, as well as lung volumes and flows. ${ }^{13}$ These variables have been commonly used to reflect respiratory function and evaluate the effectiveness of various types of interventions in people with stroke. ${ }^{14-16}$ Neuromuscular electrical stimulation, ${ }^{14}$ transcranial magnetic stimulation, ${ }^{15}$ breathing exercises (breathing/chest expansion/diaphragmatic exercises), ${ }^{16}$ and respiratory muscle training ${ }^{6}$ are examples of applied interventions, which have the potential to improve respiratory function. These interventions may increase the strength and endurance of the respiratory muscles, speed of contractions and power outputs, diaphragm thickness,

Drs Menezes, Nascimento, and Teixeira-Salmela, as well as Mr Avelino and Ms Alvarenga, are affiliated with the NeuroGroup, Department of Physiotherapy, Universidade Federal de Minas Gerais, Brazil. Dr Nascimento is also affiliated with the Center of Health Sciences, Discipline of Physiotherapy, Universidade Federal do Espírito, Santo, Brazil.

The authors disclose relationships with the following national funding agencies: Conselho Nacional de Desenvolvimento Científico e Tecnológico (CNPq, grant \# 304434/2014-0) and Fundação de Amparo à Pesquisa de Minas Gerais (FAPEMIG, PPM 00082-16).

Supplementary material related to this paper is available at http:// www.rcjournal.com.

Correspondence: Kênia KP Menezes, Department Physiotherapy, Universidade Federal de Minas Gerais, Brazil. E-mail: keniakiefer@yahoo.com.br.

DOI: $10.4187 /$ respcare. 06000 and lung volumes and flows. ${ }^{6,14-16}$ Thus, the knowledge of the most effective interventions is fundamental for professionals because this information may help them integrate the best research evidence into their clinical practice. This, when associated with clinical expertise and client preferences, will produce appropriate and effective services. ${ }^{17,18}$

There have been 4 systematic reviews that examined improvements in outcomes related to respiratory function in people with stroke, but the delivered intervention was always respiratory muscle training. $3,6,19,20$ The results indicated that respiratory muscle training resulted in increased strength of the inspiratory $\left(7 \mathrm{~cm} \mathrm{H}_{2} \mathrm{O}\right)^{6,19}$ and expiratory (13 $\mathrm{cm} \mathrm{H}_{2} \mathrm{O}$ ) muscles ${ }^{6}$ and improved lung function, such as FVC (2.0 L). ${ }^{19}$ Although numerous randomized clinical trials $^{14-16}$ have investigated the effects of other interventions aimed at improving respiratory function in people with stroke, there were no systematic reviews found that summarized the current evidence. In addition, improvements in impairments related to respiratory function have the potential to reduce activity-related symptoms, such as dyspnea, and the benefits could be carried over to everyday activities, due to more efficient use of the respiratory muscles in activities of daily living. Therefore, the effects of respiratory interventions on dyspnea and the carryover effects to activity should also be investigated.

Thus, the aim of this review was to investigate all current interventions that have been utilized to improve respiratory function and activity after stroke. The findings may help professionals select the best one. To make recommendations based on the highest level of evidence, this review included only randomized, controlled trials. ${ }^{21,22}$

\section{Methods}

\section{Focused Questions}

A systematic review was conducted to summarize all current interventions that have been utilized to improve respiratory function and activity after stroke. The specific research questions were: What are the interventions that have been delivered to improve respiratory function after stroke? Which interventions are effective in improving respiratory function after stroke? Are any benefits carried over to activity?

\section{Identification and Selection of Trials}

Searches for relevant studies, without date or language restrictions, were conducted in the following databases: CINAHL (1986 to January 2017), LILACS (1986 to January 2017), MEDLINE (1946 to January 2017), and the Physiotherapy Evidence Database (PEDro; www.pedro. org.au) (1999 to January 2017). Optimized and specific search strategies were used for all databases, by combining 
key words such as stroke and randomized controlled trials and words related to respiratory interventions, such as inspiratory muscle training, expiratory muscle training, breathing exercises, and respiratory therapy (see the supplementary materials at http://www.rcjournal.com). Title and abstracts were displayed and screened by two reviewers (KKPM, PRA), to identify relevant studies. Full-text copies of relevant peer-reviewed articles were retrieved and their reference lists were screened to identify further relevant studies. The method section of the retrieved articles was extracted and independently reviewed by KKPM and PRA, using predetermined criteria. Both reviewers were blinded to authors, journals, and results of the studies. Disagreement or ambiguities were resolved, after discussion, by consensus.

\section{Participants, Interventions, and Outcome Measures}

Trials involving adult participants $(>18$ y old) of both sexes and at any time after stroke onset were included. The number of participants, age, and time since stroke were registered for description purposes. At admission to the trial, participants who were $<6$ months after stroke were categorized as acute/sub-acute, and those who were $>6$ months after stroke were categorized as chronic.

The experimental intervention had to be planned, structured, repetitive, purposive, and delivered with the aim of improving respiratory function after stroke. All forms of active exercises (eg, aerobic, strength, breathing, and electrical stimulation) were included. Trials were excluded if the experimental interventions were one or more of the following: multidisciplinary, primarily occupational therapy, invasive procedures, drug therapy, single-session therapy, education, sensory or brain stimulation, without active exercises. Feasibility studies and study protocols were not examined. The comparisons of interest were respiratory intervention versus no intervention/sham or versus other interventions.

Trials were examined when at least one outcome related to respiratory function was measured. The primary outcomes of interest were strength measures of the respiratory muscles (eg, maximum inspiratory pressure $\left[\mathrm{P}_{\text {Imax }}\right]$, maximum expiratory pressure $\left[\mathrm{P}_{\mathrm{Emax}}\right]$, and endurance) or lung function measured via spirometry (eg, $\mathrm{FVC}, \mathrm{FEV}_{1}$, and peak expiratory flow [PEF]).

Secondary outcomes were dyspnea and activity. Dyspnea was defined as an uncomfortable abnormal awareness of breathing and had to be measured using validated self-reported scales (eg, Borg scale). The activity measurement had to be representative of the ability to execute tasks or actions. Direct measures or self-reported questionnaires were used, regardless of whether they produced continuous or categorical data. Measures of general activ- ity (eg, Barthel Index) were used if they were the only available measure of activity.

\section{Quality and Risk of Bias}

The quality of the included trials was assessed by extracting the PEDro scores from the Physiotherapy Evidence Database (www.pedro.org.au). Where a trial was not included in the database, it was independently scored by two reviewers who had completed the PEDro scale training tutorial. The PEDro is an 11-item scale designed for rating the methodological quality (internal validity and statistical information) of randomized trials. Each item, except for Item 1, contributes to 1 point to the total PEDro score (range $0-10$ points). ${ }^{23}$

Risk of bias in the included trials was also independently assessed by 2 authors (KKPM, LRN), in accordance with the Cochrane Handbook for Systematic Reviews of Interventions. ${ }^{24}$ Disagreements were resolved by reaching consensus through discussion.

\section{Data Analyses}

Information regarding the method (ie, design, participants, intervention, outcome measures) and results (ie, number of participants, and mean [SD]) of respiratory outcomes) were extracted by 2 independent reviewers and verified by a third one. When information was not available in the published trials, details were requested from the corresponding author.

To obtain the pooled estimate of the effects of the interventions, the change scores and/or post-intervention scores were extracted and analyzed using a random effects model. The pooled data for all outcomes were reported as weighted mean difference (MD) or standardized mean difference (SMD), along with their respective 95\% confidence intervals $(95 \% \mathrm{CI})$. Analyses were performed using the Comprehensive Meta-Analysis software (Version 3.0; Biostat, Englewood, New Jersey). The critical value for rejecting $\mathrm{H}_{0}$ was set at a level of 0.05 (2-tailed). Where insufficient data were available for a study result to be included in the pooled analysis, the between-group difference was reported.

\section{Results}

\section{Flow of Trials Through the Review}

The electronic search strategy identified 2,914 articles, of which 344 were duplicates. After screening titles, abstracts, and reference lists, 46 potentially relevant full articles were retrieved. However, 29 failed to meet the inclusion criteria (for a summary of the excluded articles, see the supplementary materials at http://www.rcjournal.com); therefore, 17 ar- 


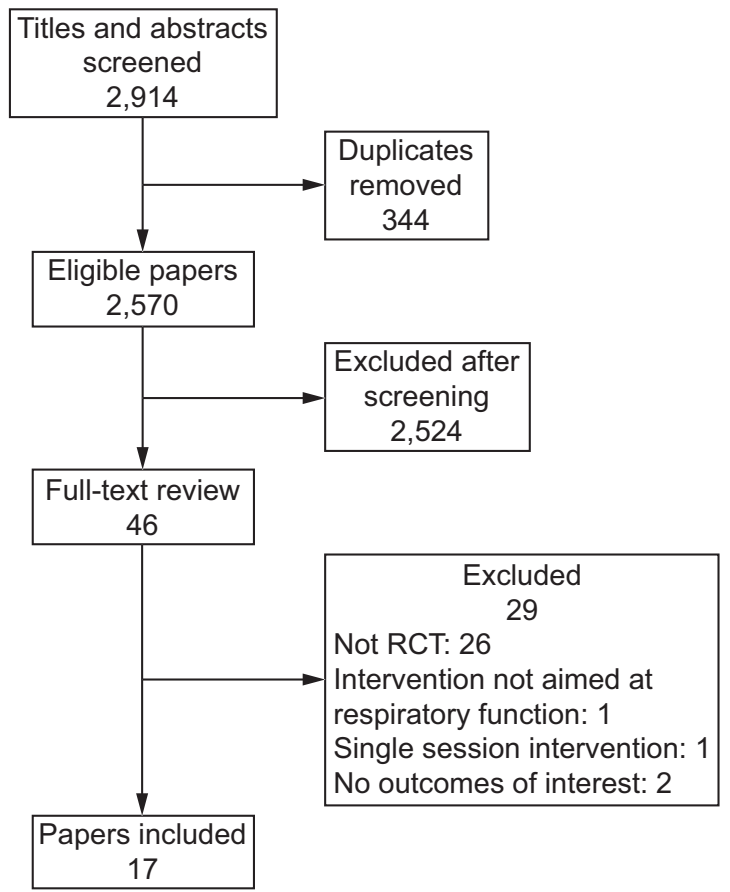

Fig. 1. Flow chart. RCT $=$ randomized controlled trial.

ticles were included in this systematic review. Figure 1 outlines the flow of the studies through the review.

\section{Characteristics of the Included Trials}

The 17 included trials involved 616 participants and investigated the effects of 5 modalities of interventions delivered to improve respiratory function after stroke. Eleven trials compared experimental interventions versus no intervention, ${ }^{14,16,25-33} 3$ compared to sham interventions, ${ }^{34-36}$ and 3 of the studies compared 2 different modalities of respiratory interventions. ${ }^{37-39}$ The characteristics of the included trials are summarized in Table 1.

Quality and Risk of Bias. The mean PEDro score of the 17 randomized included trials was 5.7 (range 4-8) (Table 2). All of the trials randomly allocated participants into groups, had similar groups at baseline, and reported between-group differences, point estimates, and variability. The majority had $<15 \%$ dropouts $(65 \%)$. On the other hand, the majority of the trials did not report blinding of assessors $(53 \%)$, concealed allocation $(65 \%)$, lacked intention-to-treat analyses $(82 \%)$, or blinded the participants $(100 \%)$ or therapists $(100 \%)$. Regarding the risk of bias, all of the included trials reported random sequence generation, and the majority (94\%) reported completed outcome data. However, allocation concealment and blinding of outcome assessment were reported in only $35 \%$ and $47 \%$ of the included trials, respectively, and none of the trials reported blinding of participants and personnel. In addition, about $80 \%$ of the included trials showed other sources of bias, such as lack of reports on intention-to-treat analysis (Fig. 2).

Participants. The mean age of the 616 participants ranged from 54 to 71 y across trials, and the mean time after stroke ranged from $9 \mathrm{~d}$ to 66 months. The majority of trials (59\%) included participants at the chronic phases after stroke.

Interventions. The experimental interventions were: aerobic exercises ( 2 trials), 37,39 breathing exercises (ie, breathing/chest expansion/diaphragmatic exercises) (4 trials), ${ }^{16,26,30,33}$ postural exercises (1 trial), ${ }^{32}$ respiratory muscle training (11 trials), ${ }^{14,25,27-31,33-36}$, and the addition of electrical stimulation ( 2 trials). ${ }^{14,38}$ Three trials included 2 interventions ( 2 experimental groups) and were included in 2 different comparisons. ${ }^{14,30,34}$ Concerning respiratory muscle training, 6 trials delivered training to the inspiratory muscles, , $^{25,27,31,33,35,36} 1$ trial to the expiratory muscles, ${ }^{35}$ and 5 trials to both inspiratory and expiratory muscles. ${ }^{14,28-30,34}$ Participants undertook training for $20-30 \mathrm{~min}$ (or 25-100 repetitions), 3-7 times per week, over 3-10 weeks.

Outcome Measures. Six trials measured strength of the respiratory muscles as maximum pressure generated during inspiration or expiration, and data were reported in $\mathrm{cm} \mathrm{H}_{2} \mathrm{O} .{ }^{14,25,33-36}$ Thirteen trials measured lung function using spirometry (data on FVC and $\mathrm{FEV}_{1}$ were reported in $\mathrm{L}$, and data on PEF were reported in $\mathrm{L} / \mathrm{s}) .{ }^{16,25-33,37-39}$ Two trials ${ }^{30,38}$ reported lung function as percentages of the predicted values. Regarding the secondary outcomes, 2 trials measured dyspnea using the Borg scale, ${ }^{29,33}$ and 6 trials measured activity. ${ }^{25,29,33,36,37,39}$ Activity was measured using timed-walk measures ( 3 trials), ${ }^{29,37,39}$ cycle ergometer (2 trials), ${ }^{33,36}$ and self-reported questionnaires (3 trials). ${ }^{25,33,36}$

\section{Effect of Interventions}

Aerobic Exercise. The effects of aerobic exercise on respiratory function were examined in 2 trials that had a mean PEDro score of 6.37,39 The first trial compared intensive with self-selected aerobic exercises, ${ }^{37}$ and the second trial compared self-selected aerobic exercises with inspiratory muscle training. ${ }^{39}$ A meta-analysis was not performed due to clinical heterogeneity. Results from the first trial $^{37}$ indicated that intensive aerobic exercises improved FVC (MD 0.4 L, 95\% CI 0.1-0.7), FEV 1 (MD 0.4 L, 95\% CI 0.1-0.7), walking speed (MD $0.1 \mathrm{~m} / \mathrm{s}, 95 \%$ CI 0.01-0.19), and walking capacity (MD $59 \mathrm{~m}, 95 \% \mathrm{CI}$ 2-116). Results from the second trial ${ }^{39}$ indicated that the 


\section{Respiratory Interventions After Stroke}

Table 1. Characteristics of the Included Randomized Controlled Trials

\begin{tabular}{|c|c|c|}
\hline Study & Participants & Intervention \\
\hline \multicolumn{3}{|l|}{ Aerobic exercises } \\
\hline Bang and Son ${ }^{37}$ & $\begin{array}{l}\text { Chronic stage } \\
N=12(7 \mathrm{men}) \\
\text { Age }(\mathrm{y})=60(6)\end{array}$ & $\begin{array}{l}\text { Experimental group: Intensive aerobic exercise with an } \\
\text { ergonomic cycle, at } 50-80 \% \text { of their maximal heart } \\
\text { rate }(30 \mathrm{~min} \times 5 / \mathrm{wk} \times 4 \mathrm{wk}) \\
\text { Control group: Self-selected intensity exercise with an } \\
\text { ergonomic cycle }(30 \text { min } \times 5 / \mathrm{wk} \times 4 \mathrm{wk})\end{array}$ \\
\hline Jung and Bang 39 & $\begin{array}{l}\text { Acute/sub-acute stages } \\
N=12(7 \text { men }) \\
\text { Age }(\mathrm{y})=62(5)\end{array}$ & $\begin{array}{l}\text { Experimental group: Inspiratory muscle training, with } \\
\text { a fixed load of } 30 \% \text { of } \mathrm{P}_{\text {Imax }}(30 \mathrm{~min} \times 5 / \mathrm{wk} \times \\
4 \mathrm{wk}+\text { conventional stroke rehabilitation program } \\
\text { or } 30 \mathrm{~min} \times 5 / \mathrm{wk} \times 4 \mathrm{wk}) \\
\text { Control group: Self-selected intensity aerobic exercise } \\
\text { with an ergonomic cycle }(30 \mathrm{~min} \times 5 / \mathrm{wk} \times 4 \mathrm{wk} \\
+ \text { conventional stroke rehabilitation program or } \\
30 \mathrm{~min} \times 5 / \mathrm{wk} \times 4 \mathrm{wk})\end{array}$ \\
\hline \multicolumn{3}{|l|}{$\begin{array}{l}\text { Breathing/chest } \\
\text { expansion/ } \\
\text { diaphragmatic } \\
\text { exercise }\end{array}$} \\
\hline Joo et $\mathrm{al}^{26}$ & $\begin{array}{l}\text { Chronic stage } \\
N=38(22 \text { men }) \\
\text { Age }(\mathrm{y})=56(10)\end{array}$ & $\begin{array}{l}\text { Experimental group: Game-based breathing exercises, } \\
\text { including } 14 \text { games, such as blowing a balloon, } \\
\text { flying a kite, an airplane, and a windmill }(25 \mathrm{~min} \times \\
3 / \mathrm{wk} \times 5 \mathrm{wk}+\mathrm{conventional} \mathrm{stroke} \mathrm{rehabilitation} \\
\text { program or } 30 \mathrm{~min} \times 5 / \mathrm{wk} \times 5 \mathrm{wk}) \\
\text { Control group: Conventional stroke rehabilitation } \\
\text { program }(30 \mathrm{~min} \times 5 / \mathrm{wk} \times 5 \mathrm{wk})\end{array}$ \\
\hline Kim et $\mathrm{al}^{30}$ & $\begin{array}{l}\text { Acute/sub-acute stages } \\
N=37(17 \text { men }) \\
\text { Age }(\mathrm{y})=59(6)\end{array}$ & $\begin{array}{l}\text { Experimental group I: Respiratory muscle training, } \\
\text { with an incentive respiratory spirometer (load not } \\
\text { reported) }(50 \mathrm{repetitions} \times 5 / \mathrm{wk} \times 6 \mathrm{wk}+ \\
\text { conventional stroke rehabilitation program or } 60 \mathrm{~min} \\
\times 5 / \mathrm{wk} \times 6 \mathrm{wk}) \\
\text { Experimental group II: Respiratory muscle training, } \\
\text { with an incentive respiratory spirometer (load not } \\
\text { reported) }(50 \text { repetitions } \times 5 / \mathrm{wk} \times 6 \mathrm{wk}+ \\
\text { abdominal drawing-in maneuver or } 50 \text { repetitions } \times \\
5 / \mathrm{wk} \times 6 \mathrm{wk}+\mathrm{conventional} \mathrm{stroke} \mathrm{rehabilitation} \\
\text { program or } 60 \mathrm{~min} \times 5 / \mathrm{wk} \times 6 \mathrm{wk}) \\
\text { Control group: Conventional stroke rehabilitation } \\
\text { program }(60 \mathrm{~min} \times 5 / \mathrm{wk} \times 6 \mathrm{wk})\end{array}$ \\
\hline Seo et $\mathrm{al}^{16}$ & $\begin{array}{l}\text { Chronic stage } \\
N=30(17 \text { men }) \\
\text { Age }(\mathrm{y})=62(3)\end{array}$ & $\begin{array}{l}\text { Experimental group: Inspiratory diaphragmatic and } \\
\text { expiratory pursed-lip breathing exercises }(15 \mathrm{~min} \times \\
5 / \mathrm{wk} \times 4 \mathrm{wk}+\text { feedback breathing device exercise } \\
\text { or } 15 \mathrm{~min} \times 5 / \mathrm{wk} \times 4 \mathrm{wk}+\text { conventional stroke } \\
\text { rehabilitation program or } 30 \mathrm{~min} \times 5 / \mathrm{wk} \times 4 \mathrm{wk}) \\
\text { Control group: Feedback breathing device exercises } \\
(15 \mathrm{~min} \times 5 / \mathrm{wk} \times 4 \mathrm{wk}+\text { conventional stroke } \\
\text { rehabilitation program or } 30 \mathrm{~min} \times 5 / \mathrm{wk} \times 4 \mathrm{wk})\end{array}$ \\
\hline Sutbeyaz et $\mathrm{al}^{33}$ & $\begin{array}{l}\text { Acute/sub-acute stages } \\
N=45(32 \mathrm{men}) \\
\text { Age }(\mathrm{y})=62(7)\end{array}$ & $\begin{array}{l}\text { Experimental group I: Inspiratory muscle training, with } \\
\text { a load of } 40 \% \text { of } \mathrm{P}_{\text {Imax }}(\text { adjusted } 5-10 \% \text { every week } \\
\text { until } 60 \% \text { of maximal strength) }(30 \mathrm{~min} \times 6 / \mathrm{wk} \times \\
6 \mathrm{wk}+\text { conventional stroke rehabilitation program- } \\
5 / \mathrm{wk} \times 6 \mathrm{wk}) \\
\text { Experimental group II: Breathing exercises }(30 \mathrm{~min} \times \\
7 / \mathrm{wk} \times 6 \mathrm{wk}+\text { conventional stroke rehabilitation } \\
\text { program-5/wk } \times 6 \mathrm{wk}) \\
\text { Control group: Conventional stroke rehabilitation } \\
\text { program }(5 / \mathrm{wk} \times 6 \mathrm{wk})\end{array}$ \\
\hline \multicolumn{3}{|l|}{ Postural exercises } \\
\hline Oh and Park ${ }^{32}$ & $\begin{array}{l}\text { Chronic stage } \\
N=37(22 \text { men }) \\
\text { Age }(\mathrm{y})=56(7)\end{array}$ & $\begin{array}{l}\text { Experimental group: Lumbar stabilization with eight- } \\
\text { step exercises }(30 \mathrm{~min} \times 3 / \mathrm{wk} \times 8 \mathrm{wk}+ \\
\text { conventional stroke rehabilitation program or } \\
30 \mathrm{~min} \times 3 / \mathrm{wk} \times 8 \mathrm{wk}) \\
\text { Control group: Conventional stroke rehabilitation } \\
\text { program }(60 \mathrm{~min} \times 3 / \mathrm{wk} \times 8 \mathrm{wk})\end{array}$ \\
\hline
\end{tabular}

Outcome Measures

Pulmonary function (spirometric variables): FVC (L), $\mathrm{FEV}_{1}$ (L)

Oxygen saturation: pulse oximeter $(\%)$

Activity: 10MWT (s) and 6MWT (m)

Pulmonary function (spirometric variables) $=$ FVC (L), FEV 1 (L)

Oxygen saturation $=$ pulse oximeter $(\%)$

Activity $=10 \mathrm{MWT}$ (seconds) and 6MWT (meters)

Pulmonary function (spirometric variables) $=$ FVC (L), $\mathrm{FEV}_{1}$ (L), MVV (L/min)

Pulmonary function (spirometric variables) $=$ $\mathrm{FVC}, \mathrm{FEV}_{1}$ (all measures were reported as $\%$ predicted)

Muscle activity $=$ costal diaphragmatic and external intercostal muscles by surface electromyography (reported as \% predicted)

Pulmonary function (spirometric variables) $=$ FVC (L), FEV 1 (L), PEF (L/s), VC (L) $\mathrm{V}_{\mathrm{T}}(\mathrm{L})$, ERV (L), IRV (L), inspiratory capacity (L)

Pulmonary function (spirometric variables) $=$ FVC (L), $\mathrm{FEV}_{1}$ (L), VC (L), PEF (L/s),

MVV (L/min), $\mathrm{FEF}_{25-75 \%}(\% \mathrm{~L})$

Strength $=\mathrm{P}_{\text {Imax }}\left(\mathrm{cm} \mathrm{H}_{2} \mathrm{O}\right)$

Dyspnea $=$ Borg Scale (6-20)

Heart hate $=$ beats $/ \mathrm{min}$

Oxygen consumption $=$ peak oxygen consumption $(\mathrm{mL} / \mathrm{kg} / \mathrm{min})$

Oxygen saturation $=$ oximeter $(\%)$

Ventilação = minute ventilation $(\mathrm{L} / \mathrm{min})$

Activity = Barthel Index (score 0-100)/Cycle ergometer (W)

Participation $=$ Medical Outcomes Study Short Form 36 (score 0-100)

Pulmonary function (spirometric variables) $=$ FVC (L), $\mathrm{FEV}_{1}$ (L), PEF (L/s) 
Table 1. Continued

\begin{tabular}{|c|c|c|c|}
\hline Study & Participants & Intervention & Outcome Measures \\
\hline \multicolumn{4}{|l|}{$\begin{array}{l}\text { Respiratory muscle } \\
\text { training }\end{array}$} \\
\hline Britto et $\mathrm{al}^{36}$ & $\begin{array}{l}\text { Chronic stage } \\
N=18(9 \text { men }) \\
\text { Age }(\mathrm{y})=54(11)\end{array}$ & $\begin{array}{l}\text { Experimental group: Inspiratory muscle training, with } \\
\text { a load of } 30 \% \text { of } \mathrm{P}_{\text {Imax }} \text { (adjusted every } 2 \mathrm{wk} \text {, } \\
\text { according the new } \mathrm{P}_{\text {Imax }} \text { value) }(30 \mathrm{~min} \times 5 / \mathrm{wk} \times \\
8 \mathrm{wk}) \\
\text { Control group: Sham inspiratory muscle training (30 } \\
\text { min } \times 5 / \mathrm{wk} \times 8 \mathrm{wk})\end{array}$ & $\begin{array}{l}\text { Strength: } \mathrm{P}_{\operatorname{Imax}}\left(\mathrm{cm} \mathrm{H}_{2} \mathrm{O}\right) \\
\text { Endurance: IME }\left(\mathrm{cm} \mathrm{H}_{2} \mathrm{O}\right) \\
\text { Activity: Human Activity Profile } \\
\quad(0-94) / \text { Cycle ergometer (W) } \\
\text { Participation: Nottingham Health Profile } \\
\quad \text { (score 0-38) }\end{array}$ \\
\hline Chen et $\mathrm{al}^{25}$ & $\begin{array}{l}\text { Acute/sub-acute stages } \\
N=21(8 \mathrm{men}) \\
\text { Age (y) }=66(13)\end{array}$ & $\begin{array}{l}\text { Experimental group: Inspiratory muscle training, with } \\
\left.\text { a load of } 30 \% \text { of } \mathrm{P}_{\text {Imax }} \text { (adjusted by } 2 \mathrm{~cm} \mathrm{H}_{2} \mathrm{O} / \mathrm{wk}\right) \\
\quad(30 \mathrm{~min} \times 5 / \mathrm{wk} \times 10 \mathrm{wk}+\text { conventional stroke } \\
\text { rehabilitation program) } \\
\text { Control group: Conventional stroke rehabilitation } \\
\text { program }\end{array}$ & $\begin{array}{l}\text { Strength: } \mathrm{P}_{\text {Imax }}, \mathrm{P}_{\text {Emax }}\left(\mathrm{cm} \mathrm{H}_{2} \mathrm{O}\right) \\
\text { Pulmonary function (spirometric variables): } \\
\text { FVC (L), FEV }(\mathrm{L}) \\
\text { Oxygen saturation: pulse oximeter }(\%) \\
\text { Perceived exertion: Borg and Fatigue } \\
\text { Assessment Scales }(0-10) \\
\text { Activity: Barthel Index }(0-100)\end{array}$ \\
\hline Guillén-Solà et $\mathrm{al}^{14}$ & $\begin{array}{l}\text { Acute/sub-acute stages } \\
N=62(38 \mathrm{men}) \\
\text { Age }(\mathrm{y})=69(9)\end{array}$ & $\begin{array}{l}\text { Experimental group I: Respiratory muscle training, } \\
\text { with a load of } 30 \% \text { of } \mathrm{P}_{\text {Imax }} \text { and } \mathrm{P}_{\text {Emax }} \text { (adjusted by } \\
\left.10 \mathrm{~cm} \mathrm{H}_{2} \mathrm{O} / \mathrm{wk}\right)(100 \mathrm{repetitions} \times 5 / \mathrm{wk} \times 3 \mathrm{wk}+ \\
\text { standard swallow therapy or } 3 \mathrm{hr} \times 5 / \mathrm{wk} \times 3 \mathrm{wk}) \\
\text { Experimental group II: Sham respiratory muscle } \\
\text { training, with a fixed load of } 10 \mathrm{~cm} \mathrm{H}_{2} \mathrm{O} / \mathrm{wk}(100 \\
\text { repetitions } \times 5 / \mathrm{wk} \times 3 \mathrm{wk}+\text { neuromuscular } \\
\text { electrical stimulation in swallow muscles or } \\
40 \mathrm{~min} \times 5 / \mathrm{wk} \times 3 \mathrm{wk}+\text { standard swallow therapy } \\
\text { or } 3 \mathrm{hr} \times 5 / \mathrm{wk} \times 3 \mathrm{wk}) \\
\text { Control group: Standard swallow therapy ( } 3 \mathrm{hr} \times \\
5 / \mathrm{wk} \times 3 \mathrm{wk})\end{array}$ & $\begin{array}{l}\text { Strength: } \mathrm{P}_{\mathrm{Imax}}, \mathrm{P}_{\mathrm{Emax}}\left(\mathrm{cm} \mathrm{H}_{2} \mathrm{O}\right) \\
\text { Dysphagia: Penetration Aspiration Scale } \\
\text { (1-8) } \\
\text { Respiratory complications: Occurrence }\end{array}$ \\
\hline Jung and $\mathrm{Kim}^{27}$ & $\begin{array}{l}\text { Chronic stage } \\
N=29(17 \text { men }) \\
\text { Age }(\mathrm{y})=59(10)\end{array}$ & $\begin{array}{l}\text { Experimental group: Inspiratory muscle training, with } \\
\text { a load of } 30 \% \text { of } \mathrm{P}_{\text {Imax }} \text { (adjusted gradually, } \\
\text { according the Borg scale })(20 \text { min } \times 3 / \mathrm{wk} \times 6 \text { wk) } \\
\text { Control group: Nothing }\end{array}$ & $\begin{array}{l}\text { Pulmonary function (spirometric variables) }= \\
\text { FVC (L), FEV } 1 \text { (L), PEF }(\mathrm{L} / \mathrm{s}) \\
\text { Diaphragm thickness: Ultrasonography }(\mathrm{cm}) \\
\text { Chest expansion: Tapeline }(\mathrm{cm})\end{array}$ \\
\hline Kim et $\mathrm{al}^{28}$ & $\begin{array}{l}\text { Chronic stage } \\
N=27(10 \mathrm{men}) \\
\text { Age }(\mathrm{y})=57(7)\end{array}$ & $\begin{array}{l}\text { Experimental group: Feedback respiratory training, } \\
\text { with the SpiroTiger device adjusted at } 50-60 \% \text { of } \\
\text { vital capacity and low frequency }(12-13 \text { breaths } / \\
\text { min })(30 \mathrm{~min} \times 3 / \mathrm{wk} \times 4 \mathrm{wk}+\text { conventional } \\
\text { stroke rehabilitation program or } 30 \text { min } \times 3 / \mathrm{wk} \times \\
4 \mathrm{wk}) \\
\text { Control group: Conventional stroke rehabilitation } \\
\text { program ( } 30 \text { min } \times 3 / \mathrm{wk} \times 4 \mathrm{wk})\end{array}$ & $\begin{array}{l}\text { Pulmonary function (spirometric variables): } \\
\text { FVC (L), FEV }(\mathrm{L}), \mathrm{PEF}(\mathrm{L} / \mathrm{s}), \mathrm{VC}(\mathrm{L}), \\
\mathrm{V}_{\mathrm{T}}(\mathrm{L}), \mathrm{ERV}(\mathrm{L}), \mathrm{IRV}(\mathrm{L}) \\
\text { Chest expansion: tapeline }(\mathrm{cm})\end{array}$ \\
\hline Kim et $\mathrm{al}^{29}$ & $\begin{array}{l}\text { Chronic stage } \\
N=20(\text { sex not reported }) \\
\text { Age }(\mathrm{y})=54(9)\end{array}$ & $\begin{array}{l}\text { Experimental group: Respiratory muscle training and } \\
\text { endurance, adjusted by the subjects' breathing } \\
\text { capacities }(20 \mathrm{~min} \times 3 / \mathrm{wk} \times 4 \mathrm{wk}+\text { conventional } \\
\text { stroke rehabilitation program or } 30 \mathrm{~min} \times 3 / \mathrm{wk} \times \\
4 \mathrm{wk}+\text { exercises using an automated full-body } \\
\text { workout machine or } 20 \mathrm{~min} \times 3 / \mathrm{wk} \times 4 \mathrm{wk}) \\
\text { Control group: Conventional stroke rehabilitation } \\
\text { program }(30 \mathrm{~min} \times 3 / \mathrm{wk} \times 4 \mathrm{wk}+\text { exercises using } \\
\text { an automated full-body workout machine or } 20 \mathrm{~min} \\
\times 3 / \mathrm{wk} \times 4 \mathrm{wk})\end{array}$ & $\begin{array}{l}\text { Pulmonary function (spirometric variables): } \\
\text { FVC (L), FEV } 1 \text { (L), PEF (L/s) } \\
\text { Activity: 6MWT (m) } \\
\text { Dyspnea: Borg Scale (1-10) }\end{array}$ \\
\hline Kim et $\mathrm{al}^{30}$ & $\begin{array}{l}\text { Acute/sub-acute stages } \\
N=37(17 \text { men }) \\
\text { Age }(\mathrm{y})=59(6)\end{array}$ & $\begin{array}{l}\text { Experimental group I: Respiratory muscle training, } \\
\text { with an incentive spirometer (load not reported) }(50 \\
\text { repetitions } \times 5 / \mathrm{wk} \times 6 \mathrm{wk}+\text { conventional stroke } \\
\text { rehabilitation program or } 60 \mathrm{~min} \times 5 / \mathrm{wk} \times 6 \mathrm{wk}) \\
\text { Experimental group II: Respiratory muscle training, } \\
\text { with an incentive spirometer (load not reported) }(50 \\
\text { repetitions } \times 5 / \mathrm{wk} \times 6 \mathrm{wk}+\text { abdominal drawing-in } \\
\text { maneuver or } 50 \mathrm{repetitions} \times 5 / \mathrm{wk} \times 6 \mathrm{wk}+ \\
\text { conventional stroke rehabilitation program or } 60 \mathrm{~min} \\
\times 5 / \mathrm{wk} \times 6 \mathrm{wk}) \\
\text { Control group: Conventional stroke rehabilitation } \\
\text { program }(60 \mathrm{~min} \times 5 / \mathrm{wk} \times 6 \mathrm{wk})\end{array}$ & $\begin{array}{l}\text { Pulmonary function (spirometric variables): } \\
\text { FVC, FEV } \text { (all measures were reported as }_{\% \text { predicted) }} \\
\text { Muscle activity: Costal diaphragmatic and } \\
\text { external intercostal muscles (surface } \\
\text { electromyography, \% predicted) }\end{array}$ \\
\hline Kulnik et al ${ }^{35}$ & $\begin{array}{l}\text { Acute/sub-acute stages } \\
N=78(47 \text { men }) \\
\text { Age }(\mathrm{y})=64(15)\end{array}$ & $\begin{array}{l}\text { Experimental group I: Inspiratory muscle training, with } \\
\text { a load of 50\% of } \mathrm{P}_{\text {Imax }} \text { (adjusted every week, } \\
\text { according the new } \mathrm{P}_{\text {Imax }} \text { value) (50 repetitions } \times \\
7 / \mathrm{wk} \times 4 \mathrm{wk} \text { ) } \\
\text { Experimental group II: Expiratory muscle training, } \\
\text { with a load of } 50 \% \text { of } \mathrm{P}_{\text {Emax }} \text { (adjusted every week, } \\
\text { according the new } \mathrm{P}_{\text {Emax value) (50 repetitions } \times} \\
7 / \mathrm{wk} \times 4 \text { wk) } \\
\text { Control group: Sham respiratory muscle training, with } \\
\text { a fixed load of } 10 \% \text { of maximum pressure (50 } \\
\text { repetitions } \times 7 / \mathrm{wk} \times 4 \mathrm{wk} \text { ) }\end{array}$ & $\begin{array}{l}\text { Strength: } \mathrm{P}_{\text {Imaax }}, \mathrm{P}_{\text {Emax }}\left(\mathrm{cm} \mathrm{H}_{2} \mathrm{O}\right) \\
\text { Cough: Peak expiratory cough flow and } \\
\text { capsaicin-induced involuntary cough } \\
\text { (L/min) } \\
\text { Respiratory complications: Occurrence of } \\
\text { pneumonia }\end{array}$ \\
\hline
\end{tabular}


Table 1. Continued

\begin{tabular}{|c|c|c|c|}
\hline Study & Participants & Intervention & Outcome Measures \\
\hline Oh et $\mathrm{al}^{31}$ & $\begin{array}{l}\text { Chronic stage } \\
N=23 \text { (13 men) } \\
\text { Age }(\mathrm{y})=71(7)\end{array}$ & $\begin{array}{l}\text { Experimental group: Inspiratory muscle training, with } \\
\text { a load of } 30 \% \text { of } \mathrm{P}_{\mathrm{Imax}}(20 \mathrm{~min} \times 3 / \mathrm{wk} \times 6 \mathrm{wk}+ \\
\text { conventional stroke rehabilitation program }+ \\
\text { abdominal strengthening and breathing exercises or } \\
\text { 3/wk } \times 6 \mathrm{wk}) \\
\text { Control group: Conventional stroke rehabilitation } \\
\text { program plus abdominal strengthening and breathing } \\
\text { exercises }(3 / \mathrm{wk} \times 6 \mathrm{wk})\end{array}$ & $\begin{array}{l}\text { Pulmonary function (spirometric variables): } \\
\text { FVC (L), FEV }(\mathrm{L}), \text { PEF }(\mathrm{L} / \mathrm{s}) \\
\text { Thickness: Transverse abdominis and internal } \\
\text { oblique muscles by ultrasonography }(\mathrm{cm}) \\
\text { Balance: Berg balance scale }(0-56)\end{array}$ \\
\hline $\begin{array}{l}\text { Messaggi-Sartor } \\
\text { et al }{ }^{44}\end{array}$ & $\begin{array}{l}\text { Acute/sub-acute stages } \\
N=109(63 \mathrm{men}) \\
\text { Age }(\mathrm{y})=67(11)\end{array}$ & $\begin{array}{l}\text { Experimental group: Respiratory muscle training, with } \\
\text { a load of } 30 \% \text { of } \mathrm{P}_{\text {Imax }} \text { and } \mathrm{P}_{\text {Emax }} \text { (adjusted } \\
10 \mathrm{~cm} \mathrm{H}_{2} \mathrm{O} \text { every week) }(100 \text { repetitions } \times 5 / \mathrm{wk} \times \\
3 \mathrm{wk}) \\
\text { Control group: Respiratory muscle training }(100 \\
\text { repetitions } \times 5 / \mathrm{wk} \times 3 \mathrm{wk})\end{array}$ & $\begin{array}{l}\text { Strength: } \mathrm{P}_{\text {Imax }}, \mathrm{P}_{\text {Emax }}\left(\mathrm{cm} \mathrm{H}_{2} \mathrm{O}\right) \\
\text { Respiratory complications: Number of lung } \\
\text { infections and pulmonary } \\
\text { thromboembolisms } \\
\text { Peripheral muscle strength: dynamometer } \\
\quad(\mathrm{kg})\end{array}$ \\
\hline Sutbeyaz et $\mathrm{al}^{33}$ & $\begin{array}{l}\text { Acute/sub-acute stages } \\
N=45(24 \text { men }) \\
\text { Age }(\mathrm{y})=62(7)\end{array}$ & $\begin{array}{l}\text { Experimental group I: Inspiratory muscle training, with } \\
\text { a load of } 40 \% \text { of } \mathrm{P}_{\text {Imax }}(\text { adjusted } 5-10 \% \text { every week } \\
\text { until } 60 \% \text { of maximal strength) }(30 \mathrm{~min} \times 6 / \mathrm{wk} \times \\
6 \mathrm{wk}+\text { conventional stroke rehabilitation program } \\
\text { or } 5 / \mathrm{wk} \times 6 \mathrm{wk}) \\
\text { Experimental group II: Breathing exercises }(30 \mathrm{~min} \times \\
7 / \mathrm{wk} \times 6 \mathrm{wk}+\text { conventional stroke rehabilitation } \\
\text { program or } 5 / \mathrm{wk} \times 6 \mathrm{wk}) \\
\text { Control group: Conventional stroke rehabilitation } \\
\text { program }(5 / \mathrm{wk} \times 6 \mathrm{wk})\end{array}$ & $\begin{array}{l}\text { Pulmonary function (spirometric variables): } \\
\text { FVC (L), FEV }(\mathrm{L}), \mathrm{VC}(\mathrm{L}), \mathrm{PEF}(\mathrm{L} / \mathrm{s}) \text {, } \\
\text { MVV (L/min), } \mathrm{FEF}_{25-75 \%}(\% \mathrm{~L}) \\
\text { Strength: } \mathrm{P}_{\text {Imax }}\left(\mathrm{cm} \mathrm{H}_{2} \mathrm{O}\right) \\
\text { Dyspnea: Borg Scale }(6-20) \\
\text { Heart rate: beats/min } \\
\text { Oxygen consumption: peak oxygen } \\
\text { consumption (mL/kg/min) } \\
\text { Oxygen saturation: oximeter }(\%) \\
\text { Ventilação: minute ventilation (L/min) } \\
\text { Functional capacity: electronically braked } \\
\text { arm crank ergometer (W) } \\
\text { Activity: Barthel Index (score 0-100) } \\
\text { Participation: Medical Outcomes Study Short } \\
\text { Form } 36 \text { (score 0-100) }\end{array}$ \\
\hline $\begin{array}{l}\text { Addition of electrical } \\
\text { stimulation }\end{array}$ & & & \\
\hline Guillén-Solà et al ${ }^{14}$ & $\begin{array}{l}\text { Acute/sub-acute stages } \\
N=62(38 \text { men }) \\
\text { Age }(\text { y) }=69(9)\end{array}$ & $\begin{array}{l}\text { Experimental group I: Respiratory muscle training, } \\
\text { with a load of } 30 \% \text { of } \mathrm{P}_{\text {Imax }} \text { and } \mathrm{P}_{\text {Emax }} \text { (adjusted by } \\
\left.10 \mathrm{~cm} \mathrm{H}_{2} \mathrm{O} / \mathrm{wk}\right)(100 \text { repetitions } \times 5 / \mathrm{wk} \times 3 \mathrm{wk}+ \\
\text { standard swallow therapy or } 3 \mathrm{hr} \times 5 / \mathrm{wk} \times 3 \mathrm{wk}) \\
\text { Experimental group II: Sham respiratory muscle } \\
\text { training, with a fixed load of } 10 \mathrm{~cm} \mathrm{H}_{2} \mathrm{O} / \mathrm{wk}(100 \\
\text { repetitions } \times 5 / \mathrm{wk} \times 3 \mathrm{wk}+\text { neuromuscular } \\
\text { electrical stimulation in swallow muscles or } \\
40 \mathrm{~min} \times 5 / \mathrm{wk} \times 3 \mathrm{wk}+\text { standard swallow therapy } \\
\text { or } 3 \mathrm{hr} \times 5 / \mathrm{wk} \times 3 \mathrm{wk}) \\
\text { Control group: Standard swallow therapy }(3 \mathrm{hr} \times 5 / \mathrm{wk} \\
\times 3 \text { wk) }\end{array}$ & $\begin{array}{l}\text { Strength: } \mathrm{P}_{\text {Imax }}, \mathrm{P}_{\text {Emax }}\left(\mathrm{cm} \mathrm{H}_{2} \mathrm{O}\right) \\
\text { Dysphagia: Penetration Aspiration Scale } \\
\quad(1-8) \\
\text { Respiratory complications: Occurrence } \\
\text { (number) }\end{array}$ \\
\hline Jung et $\mathrm{al}^{38}$ & $\begin{array}{l}\text { Chronic stage } \\
N=18(11 \text { men }) \\
\text { Age }(\mathrm{y})=55\end{array}$ & $\begin{array}{l}\text { Experimental group: Inspiratory muscle training, with } \\
\text { a fixed load of } 30 \% \text { of } \mathrm{P}_{\text {Imax }} \text {, while stimulation was } \\
\text { applied to the abdominal region on the expiration } \\
\text { moment }(20 \mathrm{~min} \times 3 / \mathrm{wk} \times 4 \mathrm{wk}) \\
\text { Control group: Inspiratory muscle training, with a } \\
\text { fixed load of } 30 \% \text { of } \mathrm{P}_{\text {Imax }}(20 \text { min } \times 3 / \mathrm{wk} \times \\
4 \mathrm{wk})\end{array}$ & $\begin{array}{l}\text { Pulmonary function (spirometric variables): } \\
\text { FVC, FEV }{ }_{1} \text {, PEF, and } \mathrm{FEF}_{25-75 \%} \text { (all } \\
\text { measures were reported as \% predicted) } \\
\text { Thickness = Diaphragm by ultrasonography } \\
\text { (not reported) }\end{array}$ \\
\hline $\begin{array}{l}10 \mathrm{MWT}=10 \text {-min walk test } \\
6 \mathrm{MWT}=6 \text {-min walk test } \\
\mathrm{P}_{\text {Imax }}=\text { maximum inspiratory } \\
\mathrm{P}_{\text {Emax }}=\text { maximum expiratory } \\
\mathrm{PEF}=\text { peak expiratory flow } \\
\mathrm{MVV}=\text { maximum voluntary } \\
\mathrm{FEF}_{25-75 \%} \text { = forced expirator } \\
\mathrm{VC}=\text { vital capacity } \\
\mathrm{V}_{\mathrm{T}}=\text { tidal volume } \\
\mathrm{ERV}=\text { expiratory reserve vo } \\
\mathrm{IRV}=\text { inspiratory reserve vol }\end{array}$ & flow between $25 \%$ and $75 \%$ of & ital capacity & \\
\hline
\end{tabular}

effects of aerobic exercise were worse than inspiratory muscle training for FVC (MD $-0.3 \mathrm{~L}, 95 \% \mathrm{CI}-0.1$ to -0.5 ) and $\mathrm{FEV}_{1}$ (MD $-0.4 \mathrm{~L}, 95 \% \mathrm{CI}-0.1$ to -0.7 ), but there was no difference between the groups for walking speed (MD $0.11 \mathrm{~m} / \mathrm{s}, 95 \% \mathrm{CI}-0.03$ to 0.25 ) and walking capacity (MD $50 \mathrm{~m}, 95 \% \mathrm{CI}-22$ to 121 ).
Breathing Exercises. The effects of breathing exercises on respiratory function were examined in 4 trials that had a mean PEDro score of 5.3. ${ }^{16,26,30,33}$ In all of these trials, the control group received no intervention. Detailed results were provided regarding the outcomes of interest. 
Table 2. PEDro Criteria and Scores of the Included Randomized Controlled Trials

\begin{tabular}{|c|c|c|c|c|c|c|c|c|c|c|c|}
\hline Study & $\begin{array}{l}\text { Random } \\
\text { Allocation }\end{array}$ & $\begin{array}{l}\text { Concealed } \\
\text { Allocation }\end{array}$ & $\begin{array}{l}\text { Groups } \\
\text { Similar at } \\
\text { Baseline }\end{array}$ & $\begin{array}{l}\text { Participant } \\
\text { Blinding }\end{array}$ & $\begin{array}{l}\text { Therapist } \\
\text { Blinding }\end{array}$ & $\begin{array}{l}\text { Assessor } \\
\text { Blinding }\end{array}$ & $\begin{array}{c}<15 \% \\
\text { Dropouts }\end{array}$ & $\begin{array}{l}\text { Intention-to- } \\
\text { Treat } \\
\text { Analysis }\end{array}$ & $\begin{array}{l}\text { Between-Group } \\
\text { Difference } \\
\text { Reported }\end{array}$ & $\begin{array}{l}\text { Point } \\
\text { Estimate and } \\
\text { Variability } \\
\text { Reported }\end{array}$ & $\begin{array}{c}\text { Total } \\
(0 \text { to } 10)\end{array}$ \\
\hline Bang and Son ${ }^{37}$ & $\mathrm{Y}$ & Y & $\mathrm{Y}$ & $\mathrm{N}$ & $\mathrm{N}$ & $\mathrm{N}$ & Y & $\mathrm{N}$ & $\mathrm{Y}$ & $\mathrm{Y}$ & 6 \\
\hline Britto et $\mathrm{al}^{36}$ & $\mathrm{Y}$ & $\mathrm{Y}$ & $\mathrm{Y}$ & $\mathrm{N}$ & $\mathrm{N}$ & $\mathrm{Y}$ & $\mathrm{Y}$ & $\mathrm{N}$ & $\mathrm{Y}$ & $\mathrm{Y}$ & 7 \\
\hline Chen et $\mathrm{al}^{25}$ & $\mathrm{Y}$ & $\mathrm{N}$ & Y & $\mathrm{N}$ & $\mathrm{N}$ & $\mathrm{Y}$ & $\mathrm{N}$ & $\mathrm{N}$ & Y & $\mathrm{Y}$ & 5 \\
\hline Guillén-Solà et al ${ }^{14}$ & $\mathrm{Y}$ & $\mathrm{N}$ & $\mathrm{Y}$ & $\mathrm{N}$ & $\mathrm{N}$ & $\mathrm{Y}$ & $\mathrm{N}$ & $\mathrm{Y}$ & $\mathrm{Y}$ & $\mathrm{Y}$ & 6 \\
\hline Joo et $\mathrm{al}^{26}$ & $\mathrm{Y}$ & $\mathrm{N}$ & $\mathrm{Y}$ & $\mathrm{N}$ & $\mathrm{N}$ & $\mathrm{N}$ & $\mathrm{Y}$ & $\mathrm{N}$ & $\mathrm{Y}$ & $\mathrm{Y}$ & 5 \\
\hline Jung and Bang 39 & Y & $\mathrm{Y}$ & $\mathrm{Y}$ & $\mathrm{N}$ & $\mathrm{N}$ & $\mathrm{N}$ & $\mathrm{Y}$ & $\mathrm{N}$ & $\mathrm{Y}$ & $\mathrm{Y}$ & 6 \\
\hline Jung and $\mathrm{Kim}^{27}$ & $\mathrm{Y}$ & $\mathrm{N}$ & $\mathrm{Y}$ & $\mathrm{N}$ & $\mathrm{N}$ & $\mathrm{Y}$ & $\mathrm{Y}$ & $\mathrm{N}$ & $\mathrm{Y}$ & $\mathrm{Y}$ & 6 \\
\hline Jung et $\mathrm{al}^{38}$ & $\mathrm{Y}$ & $\mathrm{N}$ & $\mathrm{Y}$ & $\mathrm{N}$ & $\mathrm{N}$ & $\mathrm{N}$ & Y & $\mathrm{N}$ & $\mathrm{Y}$ & $\mathrm{Y}$ & 5 \\
\hline Kim et $\mathrm{al}^{28}$ & $\mathrm{Y}$ & $\mathrm{N}$ & $\mathrm{Y}$ & $\mathrm{N}$ & $\mathrm{N}$ & $\mathrm{Y}$ & $\mathrm{N}$ & $\mathrm{N}$ & $\mathrm{Y}$ & $\mathrm{Y}$ & 5 \\
\hline Kim et $\mathrm{al}^{29}$ & $\mathrm{Y}$ & $\mathrm{N}$ & $\mathrm{Y}$ & $\mathrm{N}$ & $\mathrm{N}$ & $\mathrm{N}$ & $\mathrm{N}$ & $\mathrm{N}$ & $\mathrm{Y}$ & $\mathrm{Y}$ & 4 \\
\hline Kim et $\mathrm{al}^{30}$ & $\mathrm{Y}$ & $\mathrm{N}$ & $\mathrm{Y}$ & $\mathrm{N}$ & $\mathrm{N}$ & $\mathrm{N}$ & $\mathrm{Y}$ & $\mathrm{N}$ & $\mathrm{Y}$ & $\mathrm{Y}$ & 5 \\
\hline Kulnik et al ${ }^{35}$ & Y & $\mathrm{Y}$ & $\mathrm{Y}$ & $\mathrm{N}$ & $\mathrm{N}$ & $\mathrm{Y}$ & $\mathrm{N}$ & $\mathrm{Y}$ & $\mathrm{Y}$ & $\mathrm{Y}$ & 7 \\
\hline Messaggi-Sartor et al ${ }^{34}$ & $\mathrm{Y}$ & $\mathrm{Y}$ & $\mathrm{Y}$ & $\mathrm{N}$ & $\mathrm{N}$ & $\mathrm{Y}$ & $\mathrm{Y}$ & $\mathrm{Y}$ & $\mathrm{Y}$ & $\mathrm{Y}$ & 8 \\
\hline Oh et $\mathrm{al}^{31}$ & Y & $\mathrm{N}$ & $\mathrm{Y}$ & $\mathrm{N}$ & $\mathrm{N}$ & $\mathrm{N}$ & $\mathrm{Y}$ & $\mathrm{N}$ & $\mathrm{Y}$ & $\mathrm{Y}$ & 5 \\
\hline Oh and Park ${ }^{32}$ & $\mathrm{Y}$ & $\mathrm{N}$ & $\mathrm{Y}$ & $\mathrm{N}$ & $\mathrm{N}$ & $\mathrm{N}$ & $\mathrm{Y}$ & $\mathrm{N}$ & $\mathrm{Y}$ & $\mathrm{Y}$ & 5 \\
\hline Seo et al ${ }^{16}$ & $\mathrm{Y}$ & $\mathrm{N}$ & $\mathrm{Y}$ & $\mathrm{N}$ & $\mathrm{N}$ & $\mathrm{N}$ & $\mathrm{N}$ & $\mathrm{N}$ & $\mathrm{Y}$ & $\mathrm{Y}$ & 4 \\
\hline Sutbeyaz et $\mathrm{al}^{33}$ & $\mathrm{Y}$ & $\mathrm{Y}$ & $\mathrm{Y}$ & $\mathrm{N}$ & $\mathrm{N}$ & $\mathrm{Y}$ & $\mathrm{Y}$ & $\mathrm{N}$ & $\mathrm{Y}$ & $\mathrm{Y}$ & 7 \\
\hline
\end{tabular}

Only one trial, with a PEDro score of 7, examined the effects of breathing exercises on muscle strength after stroke (eg, maximum inspiratory pressure $\left[\mathrm{P}_{\text {Imax }}\right]$, maximum expiratory pressure $\left.\left[\mathrm{P}_{\text {Emax }}\right]\right){ }^{33}$ The mean differences between the groups were $4 \mathrm{~cm} \mathrm{H}_{2} \mathrm{O}$ (95\% CI 1-7) and $2 \mathrm{~cm} \mathrm{H}_{2} \mathrm{O}$ (95\% CI 1-4) for $\mathrm{P}_{\text {Imax }}$ and $\mathrm{P}_{\text {Emax }}$, respectively, in favor of the experimental group.

The effects of breathing exercises on FVC were examined by pooling the data from 3 trials, with a mean PEDro score of 5.3 representing moderate quality $(n=98$ participants). ${ }^{16,26,33}$ Breathing exercises did not significantly change FVC (MD $0.28 \mathrm{~L}, 95 \% \mathrm{CI}-0.04$ to $0.60, \mathrm{I}^{2}=54 \%$ ) compared with no intervention (for the detailed forest plot, see the supplementary materials at http://www.rcjournal. com). One trial, which had a PEDro score of 5, examined the effects on FVC of breathing exercises associated with respiratory muscle training compared to respiratory muscle training alone. ${ }^{30}$ Results were reported as percentages of the predicted values, and the mean difference between the groups was $4 \%$ (95\% CI 3-6) in favor of the association of breathing exercises and respiratory muscle training.

The effects of breathing exercises on $\mathrm{FEV}_{1}$ were examined by pooling the data from 3 trials, with a mean PEDro score of 5.3 representing moderate quality $(n=98$ participants). ${ }^{16,26,33}$ Breathing exercises did not significantly change $\mathrm{FEV}_{1}$ (MD $-0.01 \mathrm{~L}, 95 \% \mathrm{CI}-0.30$ to 0.28 , $\mathrm{I}^{2}=50 \%$ ) compared with no intervention (for the detailed forest plot, see the supplementary materials at http:// www.rcjournal.com). One trial, which had a PEDro score of 5, examined the effects on $\mathrm{FEV}_{1}$ of breathing exercises associated with respiratory muscle training compared to respiratory muscle training alone. ${ }^{30}$ Results were reported as percentages of the predicted values, and the mean difference between the groups was $10 \%(95 \%$ CI 8-11) in favor of the association of breathing exercises and respiratory muscle training.

The effects of breathing exercises on PEF were examined by pooling the data from 2 trials, with a mean PEDro score of 5.5 representing moderate quality $(n=60$ participants). ${ }^{16,33}$ Breathing exercises did not significantly change PEF (MD $0.21 \mathrm{~L} / \mathrm{s}, 95 \% \mathrm{CI}-0.38$ to $0.80, \mathrm{I}^{2}=0 \%$ ) compared to no intervention (for the detailed forest plot, see the supplementary materials at http://www.rcjournal. com). Two trials did not measure PEF. 26,30

Only 1 trial, which had a PEDro score of 7, examined the effects of breathing exercises on dyspnea after stroke. ${ }^{33}$ The mean between-group difference on the Borg scale was 0.1 (range $6-20,95 \%$ CI -1 to 1 ).

Only one trial, which had a PEDro score of 7, examined the effects of breathing exercises on activity. ${ }^{33}$ The mean difference between the groups was not calculated due to insufficient data, but the authors reported significantly improvement in the Barthel Index in favor of breathing exercises.

Postural Exercises. The effects of postural exercises on respiratory function compared to no intervention were examined in 1 trial, which had a PEDro score of 5.32 The results indicated that postural exercises improved $\mathrm{FVC}$ (MD 1.2 L, 95\% CI 0.6-1.8), $\mathrm{FEV}_{1}$ (MD 1.3 L, 95\% CI 

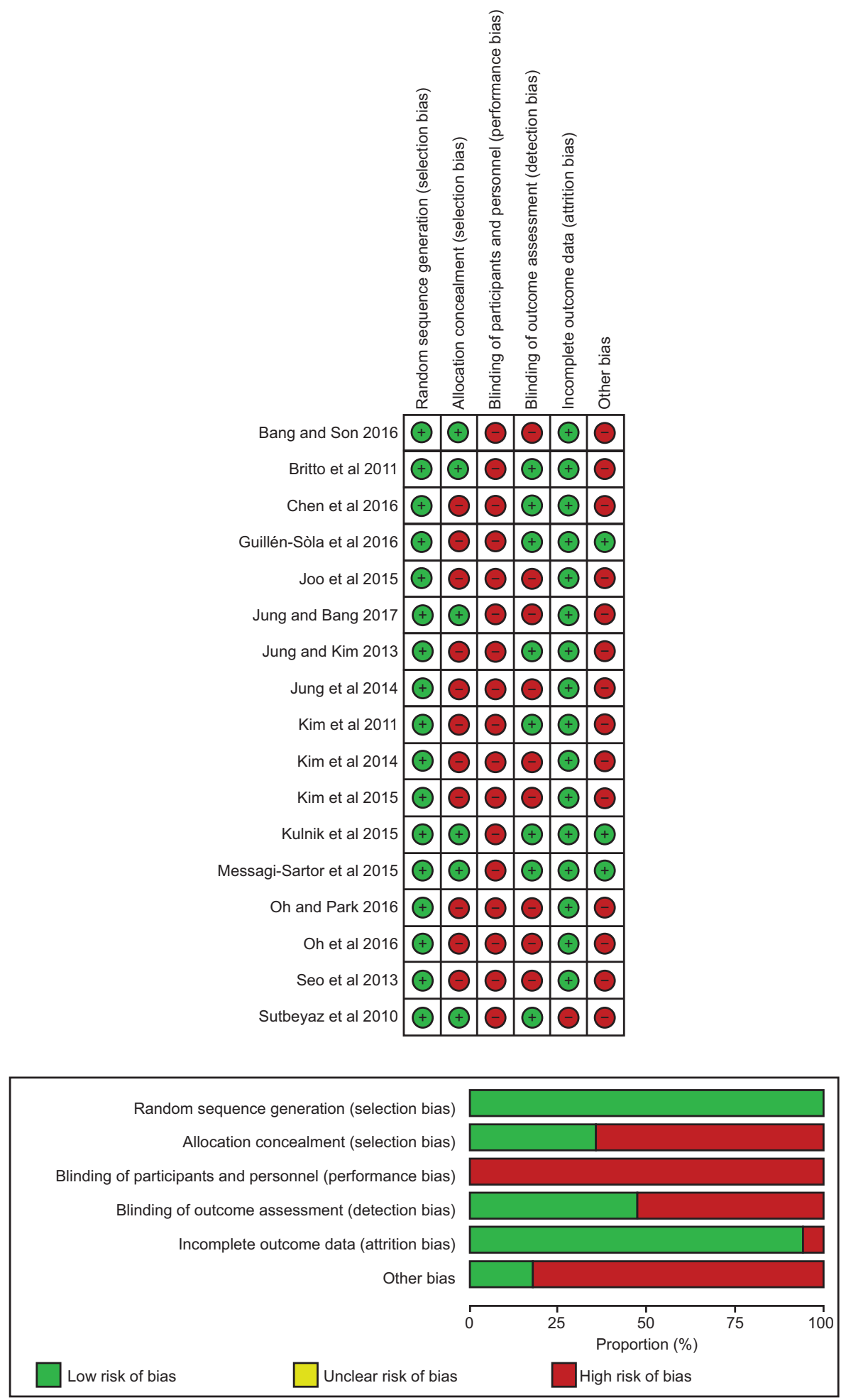

Fig. 2. Risk of bias summary and risk of bias graph: review authors' judgments about each risk of bias item for each included study. Presented as percentages across all included studies.

0.8-1.8), and PEF (MD 1.4 L, 95\% CI 0.6-2.2). The remaining outcomes of interest (ie, $\mathrm{P}_{\text {Imax }}, \mathrm{P}_{\text {Emax }}$, dyspnea, and activity) were not examined.
Respiratory Muscle Training. The effects of respiratory muscle training on respiratory function were examined in 11 trials, which had a mean PEDro score of 6. ${ }^{14,25,27-31,33-36}$ 


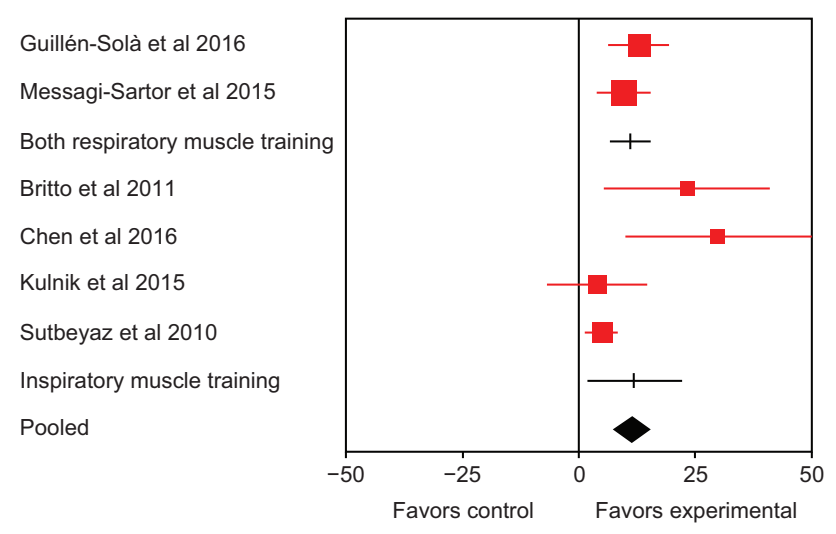

Fig. 3. Mean difference $(95 \% \mathrm{Cl})$ of the effects of respiratory muscle training vs nothing/sham respiratory intervention on strength of the inspiratory muscles, in $\mathrm{cm} \mathrm{H}_{2} \mathrm{O}(n=229)$.

The control group received no intervention ${ }^{14,25,27-31,33}$ or sham intervention. ${ }^{34-36}$ Detailed results were provided regarding the outcomes of interest.

The effects of inspiratory muscle training on strength of the inspiratory muscles $\left(\mathrm{P}_{\text {Imax }}\right)$ were examined by pooling the data from 6 trials, with a mean PEDro score of 6.7 representing moderate quality $(n=229$ participants). ${ }^{14,25,33-36}$ Overall, inspiratory muscle training increased $\mathrm{P}_{\text {Imax }}$ by $11 \mathrm{~cm} \mathrm{H}_{2} \mathrm{O}\left(95 \% \mathrm{CI} 7-15, \mathrm{I}^{2}=0 \%\right)$ compared to no intervention or sham intervention. Four trials delivered both inspiratory and expiratory muscle training (MD $11 \mathrm{~cm} \mathrm{H}_{2} \mathrm{O}, 95 \%$ CI 7-15, $\mathrm{I}^{2}=0 \%$ ), and 2 trials delivered only inspiratory muscle training (MD $12 \mathrm{~cm} \mathrm{H}_{2} \mathrm{O}$, 95\% CI 2-22, $\mathrm{I}^{2}=27 \%$ ) (Fig. 3; for the detailed forest plot, see the supplementary materials at http://www. rcjournal.com). Five trials did not measure $\mathrm{P}_{\text {Imax }} \cdot{ }^{27-31}$

The effects of expiratory muscle training on expiratory muscle strength $\left(\mathrm{P}_{\text {Emax }}\right)$ were examined by pooling the data from 3 trials ${ }^{14,34,35}(n=160$ participants $)$, with a mean PEDro score of 7.0, representing moderate quality. Overall, expiratory muscle training increased $\mathrm{P}_{\mathrm{Emax}}$ by $8 \mathrm{~cm} \mathrm{H}_{2} \mathrm{O}\left(95 \% \mathrm{CI} 2\right.$ to $\left.15 ; \mathrm{I}^{2}=65 \%\right)$; compared to nothing/sham intervention. Two trials delivered both expiratory and inspiratory muscle training (MD: $15 \mathrm{~cm}$ $\mathrm{H}_{2} \mathrm{O}, 95 \%$ CI 6 to $24 ; \mathrm{I}^{2}=0 \%$ ), and one trial delivered only expiratory muscle training (MD: $0 \mathrm{~cm} \mathrm{H}_{2} \mathrm{O}$, 95\% CI -10 to 10). (Fig. 4; for the detailed forest plot, see the supplementary materials at http://www.rcjournal. com). Six trials $27-31,36$ did not measure $\mathrm{P}_{\text {Emax }}$.

The effects of respiratory muscle training (ie, inspiratory and/or expiratory muscle training) on FVC were examined by pooling the data from 6 trials, with a mean PEDro score of 5.5 representing moderate quality $(n=150$ participants). ${ }^{25,27-29,31,33}$ Overall, respiratory muscle training increased FVC by $0.25 \mathrm{~L}\left(95 \% \mathrm{CI} 0.12-0.37, \mathrm{I}^{2}=29 \%\right)$, compared to no intervention/sham intervention. One trial delivered both inspiratory and expiratory muscle training

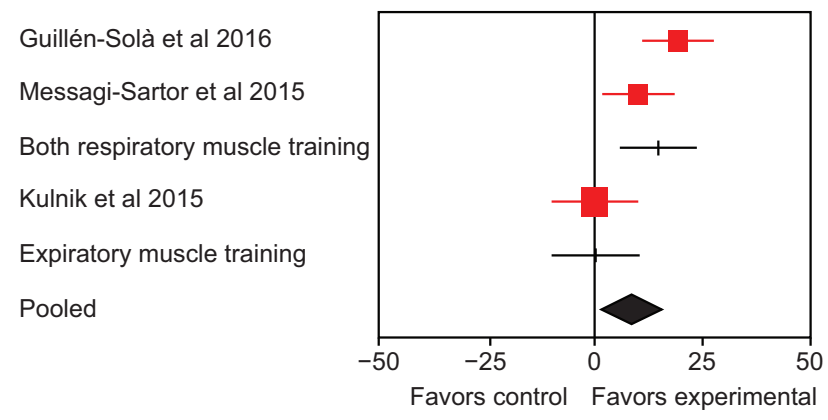

Fig. 4. Mean difference $(95 \% \mathrm{Cl})$ of the effects of respiratory muscle training vs nothing/sham respiratory intervention on strength of the expiratory muscles, in $\mathrm{cm} \mathrm{H}_{2} \mathrm{O}(n=160)$.

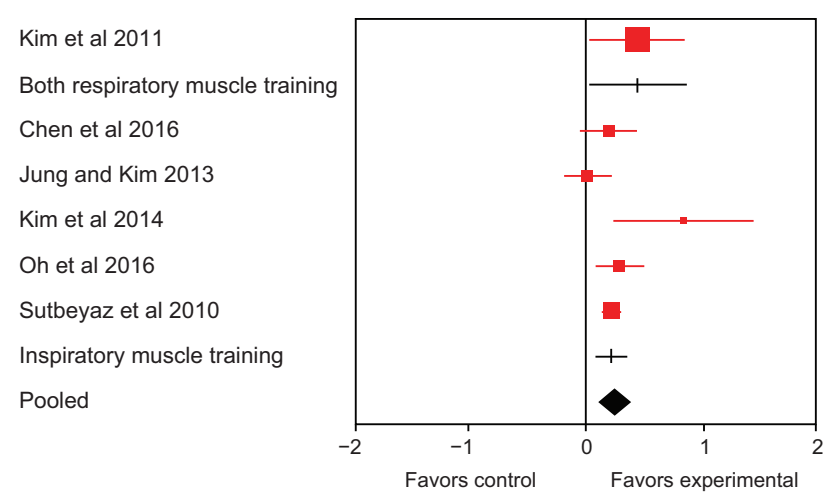

Fig. 5. Mean difference $(95 \% \mathrm{Cl})$ of the effect of respiratory muscle training vs nothing/sham respiratory intervention on forced vital capacity, in $L(n=150)$.

(MD 0.45 L, 95\% CI 0.03-0.87), and 5 trials delivered only inspiratory muscle training (MD $0.23 \mathrm{~L}, 95 \% \mathrm{CI}$ $0.09-0.36, \mathrm{I}^{2}=33 \%$ ) (Fig. 5; for the detailed forest plot, see the supplementary materials at http://www.rcjournal. com). One trial, which had a PEDro score of 5, examined the effects on FVC of respiratory muscle training versus no intervention..$^{30}$ Results were reported as percentages of the predicted values, and the mean difference between the groups was $10 \%$ (95\% CI 8-11) in favor of respiratory muscle training. Four trials did not measure FVC. ${ }^{14,34-36}$

The effects of respiratory muscle training on $\mathrm{FEV}_{1}$ were examined by pooling the data from 6 trials, with a mean PEDro score of 5.5 representing moderate quality $(n=150$ participants). ${ }^{25,27-29,31,33}$ Overall, respiratory muscle training increased $\mathrm{FEV}_{1}$ by $0.24 \mathrm{~L}\left(95 \% \mathrm{CI} 0.17-0.30, \mathrm{I}^{2}=0 \%\right)$ compared to no intervention/sham intervention. One trial delivered both inspiratory and expiratory muscle training (MD $0.36 \mathrm{~L}, 95 \% \mathrm{CI}-0.02$ to 0.74 ), and 5 trials delivered only inspiratory muscle training (MD $0.23 \mathrm{~L}, 95 \% \mathrm{CI}$ $0.17-0.30, \mathrm{I}^{2}=1 \%$ ) (Fig. 6; for the detailed forest plot, see the supplementary materials at http://www.rcjournal. com). One trial, which had a PEDro score of 5, examined the effects of respiratory muscle training versus no intervention 


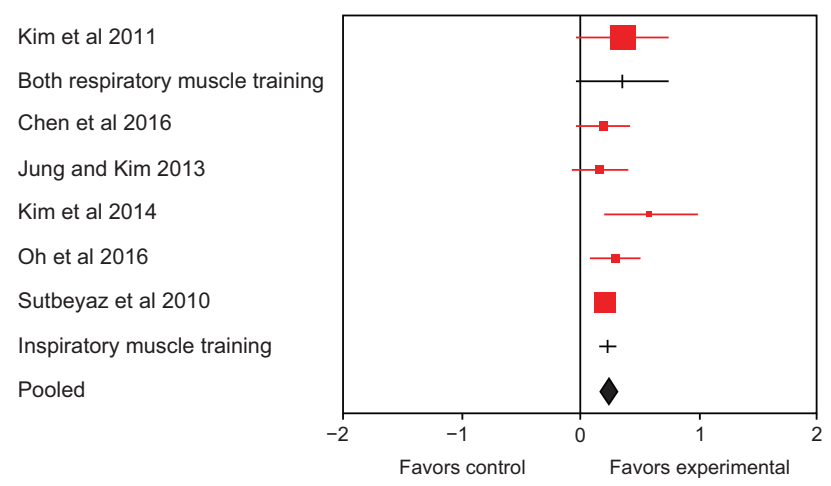

Fig. 6. Mean difference $(95 \% \mathrm{Cl})$ of the effect of respiratory muscle training vs nothing/sham respiratory intervention on $\mathrm{FEV}_{1}$, in $\mathrm{L}(n=$ 150).

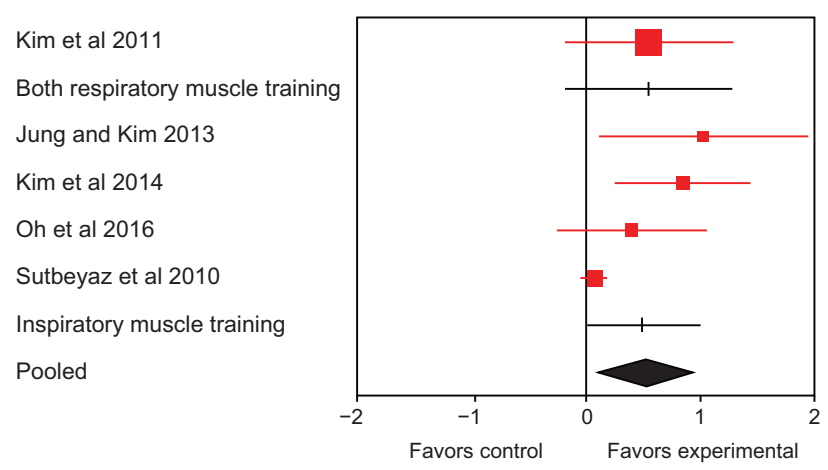

Fig. 7. Mean difference $(95 \% \mathrm{Cl})$ of the effect of respiratory muscle training vs nothing/sham respiratory intervention on peak expiratory flow, in L/s $(n=129)$.

on $\mathrm{FEV}_{1} \cdot{ }^{30}$ Results were reported as percentages of the predicted values, and the mean difference between the groups was $4 \%$ (95\% CI -1 to 9 ) in favor of respiratory muscle training. Four trials did not measure $\mathrm{FEV}_{1} \cdot{ }^{14,34-36}$

The effects of respiratory muscle training on PEF were examined by pooling the data from 5 trials, with a mean PEDro score of 5.6 representing moderate quality ( $n=129$ participants). ${ }^{27-29,31,33}$ Overall, respiratory muscle training increased PEF by $0.51 \mathrm{~L} / \mathrm{s}$ (95\% CI $0.10-$ $0.92, \mathrm{I}^{2}=0 \%$ ) compared to no intervention/sham intervention. One trial delivered both inspiratory and expiratory muscle training (MD $0.55 \mathrm{~L} / \mathrm{s}, 95 \% \mathrm{CI}-0.17$ to 1.27 ), and 4 trials delivered only inspiratory muscle training $\left(0.49 \mathrm{~L} / \mathrm{s}, 95 \% \mathrm{CI}-0.01\right.$ to $\left.0.99, \mathrm{I}^{2}=0 \%\right)$ (Fig. 7; for the detailed forest plot, see the supplementary materials at http://www.rcjournal.com). Six trials did not measure PEF. ${ }^{14,25,30,34-36}$

The effects of respiratory muscle training on dyspnea were examined by pooling the data from 2 trials, with a mean PEDro score of 5.5 representing moderate quality ( $n=50$ participants). ${ }^{29,33}$ Overall, respiratory muscle training reduced dyspnea (SMD -1.6 points, $95 \% \mathrm{CI}-2.2$ to $-0.9, \mathrm{I}^{2}=0 \%$ ) compared to no intervention/sham in- tervention (for the detailed forest plot, see the supplementary materials at http://www.rcjournal.com). Nine trials did not measure dyspnea. ${ }^{14,25,27,28,30,31,34-36}$

The effects of respiratory muscle training on activity were examined by pooling the data from 3 trials, with a mean PEDro score of 5.5 representing moderate quality ( $n=59$ participants). ${ }^{25,29,36}$ Overall, respiratory muscle training improved activity (SMD $0.78,95 \%$ CI $0.22-1.35$, $\mathrm{I}^{2}=0 \%$ ) compared to no intervention/sham intervention (for the detailed forest plot, see the supplementary materials at http://www.rcjournal.com). One trial, which had a PEDro score of 7, examined the effects inspiratory muscle training on activity, finding significant improvements in the Barthel Index in favor of the experimental group..$^{33}$ However, the mean difference between the groups could not be calculated due to insufficient data. Seven trials did not measure activity. ${ }^{14,27,28,30,31,34,35}$

The effects of the addition of electrical stimulation on respiratory function were examined in 2 trials. ${ }^{14,38}$ The first, which had a PEDro score of 6, compared electrical stimulation plus sham respiratory muscle training versus no intervention on $\mathrm{P}_{\text {Imax }}$ and $\mathrm{P}_{\text {Emax }} \cdot{ }^{14}$ The mean differences between the groups were $12 \mathrm{~cm} \mathrm{H}_{2} \mathrm{O}$ (95\% CI 3-20) and $13 \mathrm{~cm} \mathrm{H}_{2} \mathrm{O}$ (95\% CI 0.4-25) for $\mathrm{P}_{\text {Imax }}$ and $\mathrm{P}_{\text {Emax }}$, respectively, in favor of the experimental group. The second trial, which had a PEDro score of 5, compared electrical stimulation plus inspiratory muscle training versus inspiratory muscle training alone on $\mathrm{FVC}, \mathrm{FEV}_{1}$, and PEF. ${ }^{38}$ The results were reported as percentages of the predicted values, and the mean differences between the groups were $6 \%$ (95\% CI -8 to 20 ) for FVC, $15 \%$ (95\% CI -2 to 31 ) for $\mathrm{FEV}_{1}$, and $24 \%$ (95\% CI 4-43) for PEF, in favor of the experimental group. None of the trials examined the effects of electrical stimulation on dyspnea or activity.

\section{Discussion}

This review aimed to investigate all current interventions that have been applied to improve respiratory function and carryover effects to activity after stroke. Among the 17 included trials, the effects of 5 interventions on the following outcomes were investigated: strength of the inspiratory and expiratory muscles (ie, $\mathrm{P}_{\text {Imax }}$ and $\mathrm{P}_{\text {Emax }}$ ), FVC, $\mathrm{FEV}_{1}$, PEF, dyspnea, and activity. Meta-analyses were performed for the effects of breathing exercises only on $\mathrm{FVC}, \mathrm{FEV}_{1}$, and PEF, while for respiratory muscle training, meta-analyses were performed for all outcomes of interest. For the remaining interventions (ie, aerobic exercises, postural exercises, and the addition of electrical stimulation), meta-analysis was not possible.

Intensive aerobic exercises, compared with self-selected ones, were effective in improving $\mathrm{FVC}, \mathrm{FEV}_{1}$, walking speed, and walking capacity. ${ }^{37}$ However, selfselected aerobic exercises, when compared with inspira- 
tory muscle training, improved walking capacity but were worse for FVC and $\mathrm{FEV}_{1}$, and there was no difference between the groups for walking speed. ${ }^{39}$ These results indicated that the effects of self-selected aerobic exercises on respiratory function were inferior to those related to intensive aerobic exercises or inspiratory muscle training. These findings were expected to some extent, because while low-intensity continuous training improves cardiorespiratory fitness and reduces lung function declines, individuals interested in enhanced outcomes should regularly perform larger volumes of training at higher intensities. ${ }^{40,41}$ Furthermore, inspiratory muscle training is a specific intervention, with proven efficacy on measures of lung volumes and flows. ${ }^{19}$ Thus, although the results are promising regarding the effects of intensive aerobic exercises and inspiratory muscle training, they were based only on 2 studies of moderate methodological quality. Thus, further studies are necessary to allow a meta-analysis to confirm these findings.

Regarding the effects of breathing exercises on respiratory function and activity after stroke, the meta-analysis results also demonstrated no significant improvements in any of the lung function measures. These results indicated that breathing exercises, compared to no intervention, did not appear to be effective in improving $\mathrm{FVC}, \mathrm{FEV}_{1}$, and PEF. Our results were different from the results of a previous systematic review that investigated the quality of the evidence in systematic reviews and analyzed the effects of breathing exercises in individuals with COPD. ${ }^{42}$ Although 1 high-quality systematic review reported significant positive effects on breathlessness, the results were based on pooled data of only 2 randomized clinical trials. ${ }^{42}$ Thus, the authors concluded that before high-quality systematic reviews can be written and conclusions drawn, more studies are necessary. ${ }^{42}$ Similarly, this review does not provide conclusive evidence to support or refute the effects of breathing exercises on respiratory function after stroke. Thus, future trials with high-quality designs and data reporting are necessary.

Only 1 study investigated the effects of postural exercises (lumbar stabilization) on respiratory function after stroke compared to no intervention, and the results demonstrated that postural exercises improved FVC, $\mathrm{FEV}_{1}$, and PEF. ${ }^{32}$ Postural changes are known to have a possible effect on respiratory function due to decreased chest wall movements and reduced lung compliance. ${ }^{43}$ Because the lungs are positioned inside the rib cage, normal or optimal thoracic spine, rib, and scapular positioning are needed for normal breathing and full lung capacity. ${ }^{13}$ A recent study that investigated the effects of specific motor control exercises of the lumbar-pelvic musculature on respiratory function in 20 obese men reported significant improvement in respiratory function, concluding that positive respiratory effects can be obtained by prescribing these exercises ${ }^{44}$ However, similar to the results related to aerobic exercises, these results were based on only a single study of moderate methodological quality, so further studies to investigate the effects of postural exercises on respiratory function are required. Furthermore, the remaining outcomes of interest (ie, $\mathrm{P}_{\text {Imax }}, \mathrm{P}_{\text {Emax }}$, dyspnea, and activity) were not examined.

The effects of respiratory muscle training on respiratory function and activity after stroke were investigated in 11 trials, and meta-analyses were performed for all outcomes of interest. ${ }^{14,25,27-31,33-36}$ The results of this systematic review provided evidence that respiratory muscle training is effective in improving respiratory function and activity after stroke. During this intervention, individuals were asked to perform repetitive breathing exercises against an external load, using a flowdependent resistance or a pressure threshold. ${ }^{20,45}$ Because respiratory muscles respond to training stimuli by undergoing adaptations to their structure in the same manner as any other skeletal muscles, their fibers must be overloaded. ${ }^{6}$ Furthermore, weakness of the respiratory muscles is associated with restrictive ventilatory patterns and reduced lung volumes and flows. 46 Thus, strengthening of the respiratory muscles has the potential to improve lung function, as demonstrated by the meta-analysis results. In addition, abnormal respiratory function may lead to dyspnea in conditions of high effort demands as well as under low effort demands, which may interfere with the performance of daily activities. ${ }^{2,3,6,36}$ These results are important for the area of stroke rehabilitation, because reduced dyspnea and increased walking capacity have the potential to improve physical activity levels and community participation after stroke. ${ }^{47}$ Corroborating with these results, 2 previous systematic reviews investigated the effects of respiratory muscle training after stroke and reported significant improvements in inspiratory and expiratory strength, ${ }^{6,19}$ lung function, ${ }^{19}$ and exercise tolerance, ${ }^{19}$ as well as decreased respiratory complications. ${ }^{6}$ On average, $30 \mathrm{~min}$ of respiratory strength training performed 5 times per week for 5 weeks can be expected to improve respiratory function in individuals after stroke. ${ }^{6}$ However, it is important to note that, whereas the results for dyspnea and activity were significant, more studies are needed to investigate the effects of the training on these variables because the meta-analyses were based on only 2 and 3 trials, respectively, which were all of moderate methodological quality.

Finally, the effects of the addition of electrical stimulation to respiratory muscle training, compared to no intervention, on respiratory function were examined in 
2 trials. ${ }^{14,38}$ Although the first study found significant results for improved respiratory muscle strength in the experimental group provided with electrical stimulation plus sham respiratory muscle training, the control group received no intervention. ${ }^{14}$ This limits conclusions regarding the efficacy of electrical stimulation associated with respiratory muscle training. One could argue that, because a sham training was applied, the gains could be attributed to the effects of electrical stimulation. However, the sham training was delivered at a fixed load of $10 \mathrm{~cm} \mathrm{H}_{2} \mathrm{O}$, which could have the potential to improve respiratory function. On the other hand, the second trial applied electrical stimulation plus inspiratory muscle training, compared to the standard inspiratory muscle training without additional intervention, but the results were not significant for any of the lung function measures except PEF. ${ }^{38}$ In contrast with these results, a recent systematic review based on data from 14 trials investigated the evidence surrounding the use of abdominal electrical stimulation on respiratory function after spinal cord injury. ${ }^{48}$ Although functional electrical stimulation was effective in improving respiratory function, the authors emphasized that further randomized, controlled trials, with larger samples and standardized protocols, were needed to fully establish the clinical efficacy of this interventions. Thus, the present results, which are based on only 2 trials of moderate methodological quality, cannot affirm the effects of electrical stimulation on respiratory function after stroke. Furthermore, none of the trials examined its effects on dyspnea or measures of activity.

This review has both strengths and limitations. This is the first review to investigate all current interventions that have been utilized to improve respiratory function and activity after stroke. In addition, the majority of the outcome measures were reported in similar units (eg, maximum respiratory pressures in $\mathrm{cm}_{2} \mathrm{O}, \mathrm{FVC}$ and $\mathrm{FEV}_{1}$ in $\mathrm{L}$, and PEF in L/s. This is unusual in rehabilitation studies. Furthermore, publication bias inherent to systematic reviews was avoided by including only randomized clinical trials published in a variety of languages, not just in English. Finally, all of the studies included in this review are recent publications (20102017), which increases the quality of the results because respiratory muscle training is a relatively new intervention for this population. However, the mean PEDro score of the 17 included trials was 5.7, which is considered to be moderate. However, it is important to note that, because it is not usually possible to blind therapists or participants on such complex interventions, the maximum score to be reached would be 8 . Other sources of bias were lack of reporting whether an intention-to-treat analysis was undertaken and the absence of concealed allocation and blinding of the assessors by the majority of the trials. Additionally, the number of participants per group (range 6-39) was quite low, opening the results to small-trial bias. Heterogeneity was also high in 3 of the performed meta-analyses (>50\%).

\section{Conclusion}

This systematic review reported 5 possible interventions intended to improve respiratory function after stroke. However, there is no evidence on the efficacy of aerobic, breathing, and postural exercises, or of the addition of electrical stimulation. Conversely, respiratory muscle training had significant evidence supporting its use in current clinical practice, with proven effects on strength of the inspiratory and expiratory muscles $\left(\mathrm{P}_{\text {Imax }}, \mathrm{P}_{\mathrm{Emax}}\right)$, lung function (FVC, $\left.\mathrm{FEV}_{1}, \mathrm{PEF}\right)$, dyspnea, and activity. Thus, although we found several interventions that may potentially improve respiratory function after stroke, further randomized, controlled trials with larger sample sizes and standardized protocols are needed to full establish clinical efficacy.

\section{REFERENCES}

1. Mozaffarian D, Benjamin EJ, Go AS, Arnett DK, Blaha MJ, Cushman M, et al. Heart disease and stroke statistics-2015 update: a report from the American Heart Association. Circulation 2015;131: e29-e322.

2. Teixeira-Salmela LF, Parreira VF, Britto RR, Brant TC, Inácio EP, Alcântara TO, et al. Respiratory pressures and thoracoabdominal motion in community-dwelling chronic stroke survivors. Arch Phys Med Rehabil 2005;86(10):1974-1978.

3. Pollock RD, Rafferty GF, Moxham J, Kalra L. Respiratory muscle strength and training in stroke and neurology: a systematic review. Int J Strok 2013;8(2):124-130.

4. Lanini B, Bianchi R, Romagnoli I, Coli C. Chest wall kinematics in patients with hemiplegia. Am J Respir Crit Care Med 2003;168(1): 109-113.

5. Jandt SR, Caballero RMS, Júnior LAF, Dias AS. Correlation between trunk control respiratory muscle strength and spirometry in patients with stroke: an observational study. Physiother Res Int 2011; 16(4):218-224.

6. Menezes KKP, Nascimento LR, Ada L, Polese JC, Avelino PR, Teixeira-Salmela LF. Respiratory muscle training increases respiratory muscle strength and reduces respiratory complications after stroke: a systematic review. J Physiother 2016;62(3):138-144.

7. De Troyer A, Zegers De Beyl D, Thirion M. Function of respiratory muscles in acute hemiplegia. Am Rev Respir Dis 1981;123(6):631632.

8. Khedr EM, El Shinawy O, Khedr T, Abdel Aziz Ali Y, Awad EM. Assessment of corticodiaphragmatic pathway and pulmonary function in acute ischemic stroke patients. Eur J Neural 2000;7(5):323330.

9. Katzan IL, Cebul RD, Husak SH, Dawson NV, Baker DW. The effect of pneumonia on mortality among patients hospitalized for acute stroke. Neurology 2003;60(4):620-625.

10. Billinger SA, Coughenour E, MacKay-Lyons MJ, Ivey FM. Reduced cardiorespiratory fitness after stroke: biological consequences and exercise-induced adaptations. Stroke Res Treat 2012;2012:1-11.

11. Rochester CL, Mohsenin V. Respiratory complications of stroke. Semin Respir Crit Care Med 2002;23(3):248-260. 
12. Bott J, Blumenthal S, Buxton M, Ellum S, Falconer C, Garrod R. Guidelines for the physiotherapy management of the adult, medical, spontaneously breathing patient. Thorax 2009;64(Suppl 1):i1-i51.

13. McConnell A. Respiratory muscle training: Theory and practice. London: Churchill Livingstone; 2013.

14. Guillén-Solà A, Messagi Sartor M, Bofill Soler N, Duarte E, Barrera MC, Marco E. Respiratory muscle strength training and neuromuscular electrical stimulation in subacute dysphagic stroke patients: a randomized controlled trial. Clin Rehabil 2017;31(6):761-771.

15. Harraf F, Ward K, Man W, Rafferty G, Mills K, Polkey M, et al. Transcranial magnetic stimulation study of expiratory muscle weakness in acute ischemic stroke. Neurology 2008;71(24):2000-2007.

16. Seo KC, Lee HM, Kim HA. The effects of combination of inspiratory diaphragm exercise and expiratory pursed-lip breathing exercise on pulmonary functions of stroke patients. J Phys Ther Sci 2013; 25(3):241-244.

17. Moher D, Liberati A, Tetzlaff J, Altman D, the PRISMA Group. Preferred reporting items for systematic reviews and meta-analyses: The PRISMA statement. PLoS Med 21;6:e1000097.

18. Thomas B, Ciliska D, Dobbins M, Micucci S. A process for systematically reviewing the literature: providing the research evidence for public health nursing interventions. Worldviews Evid Based Nurs 2004;1(3):176-184.

19. Gomes-Neto M, Saquetto MB, Silva CM, Carvalho VO, Ribeiro N, Conceição CS. Effects of respiratory muscle training on respiratory function, respiratory muscle strength, and exercise tolerance in patients post stroke: a systematic review with meta-analysis. Arch Phys Med Rehabil 2016;97(11):1994-2001.

20. Xiao Y, Luo M, Wang J, Luo H. Inspiratory muscle training for the recovery of function after stroke. Cochrane Database Syst Rev 2012; 16(5):CD009360.

21. Padula R, Pires R, Alouche S, Chiavegato L, Lopes A, Costa LOP. Analysis of reporting of systematic reviews in physical therapy published in Portuguese. Braz J Phys Ther 2012;16(4):281-289.

22. Herbert R, Jamtvedt G, Mead J, Hagen K. Practical evidence-based physiotherapy. London: Churchill Livingstone; 2011.

23. Maher CG, Sherrington C, Herbert RD, Moseley AM, Elkins M. Reliability of the PEDro scale for rating quality of randomized controlled trials. Phys Ther 2003;83(8):713-721.

24. Higgins JPT, Green S (eds). Cochrane Handbook for Systematic Reviews of Interventions, Version 5.1.0 [updated Mar 2011]. The Cochrane Collaboration, 2011. Available at http://handbook. cochrane.org.

25. Chen PC, Liaw MY, Wang LY, Tsai YC, Hsin YJ, Chen YC, et al. Inspiratory muscle training in stroke patients with congestive heart failure: a CONSORT-compliant prospective randomized single-blind controlled trial. Medicine (Baltimore) 2016;95(37):e4856.

26. Joo S, Shin D, Song C. The effects of game-based breathing exercise on pulmonary function in stroke patients: a preliminary study. Med Sci Monit 2015;21:1806-1811.

27. Jung JH, Kim NS. Effects of inspiratory muscle training on diaphragm thickness, pulmonary function, and chest expansion in chronic stroke patients. J Korean Soc Phys Med 2013;8(1):59-69.

28. Kim K, Fell DW, Lee JH. Feedback respiratory training to enhance chest expansion and pulmonary function in chronic stroke: a doubleblind, randomized controlled study. J Phys Ther Sci 2011;23(1): 75-79.

29. Kim J, Park JH, Yim J. Effects of respiratory muscle and endurance training using an individualized training device on the pulmonary function and exercise capacity in stroke patients. Med Sci Monit 2014;20:2543-2549.

30. Kim CY, Lee JS, Kim HD, Kim IS. Effects of the combination of respiratory muscle training and abdominal drawing-in maneuver on respiratory muscle activity in patients with post-stroke hemiplegia: a pilot randomized controlled trial. Top Stroke Rehabil 2015;22(4): 262-270.

31. Oh D, Kim G, Lee W, Shin MMS. Effects of inspiratory muscle training on balance ability and abdominal muscle thickness in chronic stroke patients. J Phys Ther Sci 2016;28(1):107-111.

32. Oh D-S, Park S-E. The effect of lumbar stabilization exercise on the pulmonary function of stroke patients. J Phys Ther Sci 2016;28(6): 1896-1900.

33. Sutbeyaz ST, Koseoglu F, Inan L, Coskun O. Respiratory muscle training improves cardiopulmonary function and exercise tolerance in subjects with subacute stroke: a randomized controlled trial. Clin Rehabil 2010;24(3):240-250.

34. Messaggi-Sartor M, Guillen-Sola A, Depolo M, Duarte E, Rodríguez DA, Barrera MC, et al. Inspiratory and expiratory muscle training in subacute stroke: a randomized clinical trial. Neurology 2015;85(7): 564-572.

35. Kulnik ST, Birring SS, Moxham J, Rafferty GF, Kalra L. Does respiratory muscle training improve cough flow in acute stroke? Pilot randomized controlled trial. Stroke 2015;46(2):447-453.

36. Britto RR, Rezende NR, Marinho KC, Torres JL, Parreira VF, Teixeira-Salmela LF. Inspiratory muscular training in chronic stroke survivors: a randomized controlled trial. Arch Phys Med Rehabil 2011; 92(2):184-190.

37. Bang D-H, Son Y-L. Effect of intensive aerobic exercise on respiratory capacity and walking ability with chronic stroke patients: a randomized controlled pilot trial. J Phys Ther Sci 2016;28(8):2381-2384.

38. Jung JH, Shim JM, Kwon HY, Kim HR, Kim BI. Effects of abdominal stimulation during inspiratory muscle training on respiratory function of chronic stroke patients. J Phys Ther Sci 2014;26(1):73-76.

39. Jung KM, Bang DH. Effect of inspiratory muscle training on respiratory capacity and walking ability with subacute stroke patients: a randomized controlled pilot trial. J Phys Ther Sci 2017;29(2):336-339.

40. Billat VL. Interval training for performance: a scientific and empirical practice: special recommendations for middle- and long-distance running. Part I: aerobic interval training. Sports Med 2001;31(1):13-31.

41. Seiler S, Joranson K, Olesen BV, Hetlelid KJ. Adaptations to aerobic interval training: interactive effects of exercise intensity and total work duration. Scand J Med Sci Sports 2013;23(1):74-83.

42. Borge CR, Hagen KB, Mengshoel AM, Omenaas E, Moum T, Wahl AK. Effects of controlled breathing exercises and respiratory muscle training in people with chronic obstructive pulmonary disease: results from evaluating the quality of evidence in systematic reviews. BMC Pulm Med 2014;14:184.

43. Szopa A, Domagalska-Szopa M. Correlation between respiratory function and spine and thorax deformity in children with mild scoliosis. Medicine (Baltimore) 2017;96(22):e7032.

44. Bezzoli E, Andreotti D, Pianta L, Mascheroni M, Piccinno L, Puricelli $\mathrm{L}$, et al. Motor control exercises of the lumbar-pelvic region improve respiratory function in obese men: a pilot study. Disabil Rehabil 2018;40(2):152-158

45. Elkins M, Dentice R. Inspiratory muscle training facilitates weaning from mechanical ventilation among patients in the intensive care unit: a systematic review. J Physiother 2015;61(3):125-134.

46. Estenne M, Gevenois PA, Kinnear W, Soudon P, Heilporn A, De Troyer A. Lung volume restriction in patients with chronic respiratory muscle weakness: the role of microatelectasis. Thorax 1993; 48(7):698-701.

47. Michaelsen SM, Ovando AC, Romaguera F, Ada L. Effect of backward treadmill training on walking capacity after stroke: a randomized clinical trial. Int J Stroke 2014;9(4):529-532.

48. McCaughey EJ, Borotkanics RJ, Gollee H, Folz RJ, McLachlan AJ. Abdominal functional electrical stimulation to improve respiratory function after spinal cord injury: a systematic review and metaanalysis. Spinal Cord 2016;54(9):628-639. 